\title{
WestVirginiaUniversity
}

THE RESEARCH REPOSITORY @ WVU

Graduate Theses, Dissertations, and Problem Reports

2001

\section{Chords of longest circuits of graphs}

\author{
Xuechao Li \\ West Virginia University
}

Follow this and additional works at: https://researchrepository.wvu.edu/etd

\section{Recommended Citation}

$\mathrm{Li}$, Xuechao, "Chords of longest circuits of graphs" (2001). Graduate Theses, Dissertations, and Problem Reports. 1450.

https://researchrepository.wvu.edu/etd/1450

This Dissertation is protected by copyright and/or related rights. It has been brought to you by the The Research Repository @ WVU with permission from the rights-holder(s). You are free to use this Dissertation in any way that is permitted by the copyright and related rights legislation that applies to your use. For other uses you must obtain permission from the rights-holder(s) directly, unless additional rights are indicated by a Creative Commons license in the record and/ or on the work itself. This Dissertation has been accepted for inclusion in WVU Graduate Theses, Dissertations, and Problem Reports collection by an authorized administrator of The Research Repository @ WVU.

For more information, please contact researchrepository@mail.wvu.edu. 


\title{
Chords of Longest Circuits of Graphs
}

\author{
by
}

\section{Xuechao Li}

A DISSERTATION

Submitted to the Eberly College of Arts and Sciences

Of

West Virginia University

in partial fulfillment of the requirements

for the degree of

\section{Doctor of Philosophy}

in

Mathematics

Dr. C.Q. Zhang (Committee Chair)

Dr.John Goldwasser

Dr. Jerzy Wojciechowski

Dr. Hong-Jian Lai

Dr. Elaine Eschen

Morgantown, West Virginia, 2001

Keywords: Chords,Projective plane,torus

Copyright 2001 Xuechao Li 


\section{ABSTRACT \\ Xuechao Li \\ Adviser: Dr. Cun-Quan Zhang}

This thesis is on a long standing open conjecture proposed by one of the most prominent mathematicians, Dr. C. Thomassen, - Every longest circuit of 3-connected graph has a chord. In 1987, C. Q. Zhang proved that every longest circuit of a 3-connected planar graph $G$ has a chord if $G$ is cubic or if the minimun degree is at least 4. In 1997, Carsten Thomassen proved that every longest circuit of 3-connected cubic graph has a chord.

In this dissertation, we prove the following three independent partial results:

(1) Every longest circuit of a 3-connected graph embedded in a projective plane with minimun degree is at least has a chord ( Theorem 2.3.1).

(2) Every longest circuit of a 3-connected cubic graph has at least two chords. Furthermore if the graph is also a planar, then every longest circuit has at least three chords (Theorem 3.2.6, 3.2.7).

(3) Every longest circuit of a 4-connected graph embedded in a torus or Klein bottle has a chord.

We get these three independent results with three totally different approaches: Connectivity (Tutte circuit), second Hamilton circuit, and charge and discharge methods. 


\section{Acknowledgements}

I would like to thank my supervisor, Dr. C. Q. Zhang, for his guidance, advice, and continual encouragement. It is my pleasure to work under his supervision. Without him, this dissertation could not have come out.

I would also like to thank other committee members: Dr. Hong-Jian. Lai, Dr. J. Wojciechowski, Dr. J. Goldwasser, and Dr. Elaine M. Eschen, for their help during my studies.

And finally, I thank my family: my parents Weizheng and Qingyi, for their constant encouragement and taking care of my son; and my son Hailong, for helping me keep a good perspective on life. 


\section{Dedication}

To

my parents

and

my son 


\section{Contents}

1 Introduction 1

1.1 Chords . . . . . . . . . . . . . . . . . . . . . . 1

1.2 Notations and Definitions . . . . . . . . . . . . . 2

2 Chords of Longest Circuits in Projective Graphs 4

2.1 Introduction . . . . . . . . . . . . . . . . 4

2.2 Definitions and lemmas . . . . . . . . . . . . . . 5

2.2 .1 Tutte circuits . . . . . . . . . . . . . 5

2.2 .2 Cross caps . . . . . . . . . . . . . . . . 5 5

2.3 Main results . . . . . . . . . . . . . . . . . . . . . . 9

3 Multiple Chords of Longest Circuits in 3-Connected Graphs 16

3.1 Introduction . . . . . . . . . . . . . . . . 16

3.2 Multiple chords of longest circuits in cubic graphs . . . . . . 17

4 Chords of Graphs Embedded in the Torus and Klein Bottle 24

4.1 Introduction and Terminology . . . . . . . . . . . . . . 24

4.2 Euler Contribution . . . . . . . . . . . . . . 26

4.3 Lemmas and Definitions . . . . . . . . . . . . . 27 
4.4 Proof of the main theorem . . . . . . . . . . . . . . 31

4.5 Remarks .................... . . 55 


\section{Chapter 1}

\section{Introduction}

\subsection{Chords}

In this dissertation, we study Thomassen's conjecture that every longest circuit of a 3-connected graph has a chord.

It is well known that not all 3-connected planar graphs are Hamiltonian. Tutte(1946) gave an example with 46 vertices of a 3-connected cubic planar graph with no Hamiltonian circuit. Also, Tutte proved that every 4-connected planar graph has a Hamilton cycle by using the Tutte's lemma (known as Tutte-circuit-lemma):

In 1987, as a part of his Ph.D dissertation, C. Q. Zhang used Tutte's Lemma ( Lemma 2.2.1) to prove that any longest circuit of a 3-connected planar graph $G$ has a chord if $G$ is cubic or $\delta \geq 4$.

Using an extension of Tutte's Lemma we prove that every longest circuit in a 3-connected graph embedded in a projective 
plane has a chord if $\delta \geq 4$.

In 1997, using Herbert Fleischner's 3-colors theorem and second Hamilton circuit technique, Carsten Thomassen proved that every longest circuit in a 3-connected cubic graph has a chord. Using Thomassen's technique, we prove that every longest circuit of a 3-connected cubic graph has at least two chords. Furthermore if the graph is also planar, then every longest circuit has at least three chords.

Using Euler edge contribution and edge charge-discharge methods, We prove that every longest circuit of a 4-connected graph embedded in a torus or Klein bottle has a chord.

\subsection{Notations and Definitions}

We consider finite simple graph with no loops or multiple edges. For a graph $G=(V, E)$, we use $V(G)$ and $E(G)$ to denote the vertex set and the edge set, respectively. An edge $e$ is called a chord of a circuit if $e$ is not an edge of the circuit and both endvertices of $e$ are on the circuit.

Let $P$ be a subgraph of a graph $G$. A $P$-bridge of $G$ is either an edge of $G \backslash E(P)$ with both ends on $P$ or a subgraph of $G$ induced by the edges in a component of $G \backslash V(P)$ and all edges from that component to $P$. For a $P$-bridge $B$ of $G$, the vertices in $B \cap P$ are the attachments of $B$ (on $\mathrm{P}$ ) denoted by $\mathrm{A}(\mathrm{B})$ and $V(B)$ is the set of vertices of the bridge $B$ (excluding 
the attachment vertices on $P$ ).

A vertex cut of a graph $G$ is a set $S \subset V(G)$ such that $G-S$ has more than one component.

A graph $G$ is $k$-connected if every vertex cut has at least $k$ vertices.

A graph $G$ is said to be planar if it can be represented on a plane in such a fashion that the vertices are all distinct points, the edges are simple curves, and no two edges meet one another except at their terminals.

Notation: If $H$ is a graph, then $H$ is also used to denote $V(H)$. 


\section{Chapter 2}

\section{Chords of Longest Circuits in Projective Graphs}

\section{$2.1 \quad$ Introduction}

As we indicated in chapter 1 , by studing the conjecture proposed by Thomassen that every longest circuit of a 3-connected graph has a chord, we get three independent partial results. In this chapter, we are going to discuss the first result:

If $G$ is a 3 -connected graph with $\delta \geq 4$ which can be embedded in a projective plane, then every longest circuit in $G$ has a chord.

A projective plane is a hemisphere, with all antipodal points on the equator identified.(A pair of diametrically opposite points on a sphere is called a pair of antipodal points.) 


\subsection{Definitions and lemmas}

\subsubsection{Tutte circuits}

Let $G$ be a graph embedded in a surface and let $C$ be a circuit of $G$ and $\mathcal{F}$ be a set of faces incident with $C$. Then $C$ is an $\mathcal{F}$-Tutte circuit if every $C$-bridge of $G$ contains at most three attachments and if every $C$-bridge of $G$ containing an edge of some member of $\mathcal{F}$ has at most two attachments.

Lemma 2.2.1 (Tutte's Lemma) Let $G$ be a 2-connected planar graph, let $e_{1}$ be an edge of $G$, let $F_{1}$ and $F_{2}$ be the two faces incident with $e_{1}$ and let $e_{2}$ be an edge on the boundary of $F_{1}$ and adjacent with $e_{1}$. Then there is an $\left\{F_{1}, F_{2}\right\}$-Tutte circuit $C_{T}$ containing both $e_{1}$ and $e_{2}$.

Lemma 2.2.2 (Thomas and $\mathrm{Yu}[12]$ ) Let $G$ be a 2-connected graph embedded in a projective plane, let $R$ be a face of $G$, and let $e \in E(R)$. Then there exists an $\{R\}$-Tutte circuit $C_{T}$ in $G$ such that

(i) $e \in E\left(C_{T}\right)$ and

(ii) every $C_{T}$-bridge that contains a non-contractible circuit is edge-disjoint from $R$.

\subsubsection{Cross caps}

A projective plane $\mathcal{P}$ is represented by a closed disk, along whose boundary, points at the ends of each diameter are identical points. After identification of diagonally opposite points, the boundary is a closed non-contractible curve $C$ of the projective plane $\mathcal{P}$. The projective plane $\mathcal{P}$ with the boundary $C$ 
is denoted by $\mathcal{P}_{C}$.

Note that we distinguish between the projective plane $P$ and one of its representatives, the cross cap $\mathcal{P}_{C}$ whose boundary is a given closed noncontractible curve $C$.

For a graph $G$ embedded in a projective plane $P$, the embedding is denoted by $\pi$. Note that for a non-contractible circuit $C=v_{1} v_{2} \cdots v_{r} v_{1}$ of $G$, the boundary of the cross cap in the embedding $\pi_{C}$ is a "circuit" $=v_{1}^{\prime} \cdots v_{r}^{\prime} v_{1}^{\prime \prime}$ $\cdots v_{r}^{\prime \prime} v_{1}^{\prime}$ where $v_{i}^{\prime}$ and $v_{i}^{\prime \prime}$ are the same vertex of the graph $G$.

Lemma 2.2.3 Let $G$ be a graph with an embedding $\pi$ in a projective plane. Let $C$ be a closed non-contractible curve of $P$. Then the projective plane $P$ has a representation $P_{C}$ with $C$ as its boundary, and the embedding $\pi$ of $G$ remains the same in $P$.

Lemma 2.2.4 If there is an embedding of $G$ in $\mathcal{P}_{C}$ such that there is only one edge of $G$ passing the boundary $C$ of cross cap, or the intersection of $G$ and $C$ consists of a single vertex of $G$, then $G$ is planar.

Lemma 2.2.5 Let $G$ be a 3-connected graph with an embedding $\pi$ in a projective plane. Assume that $G$ has a 3-edge-cut $T$ and $Q_{1}, Q_{2}$ as the components of $G \backslash T$. For each $\{i, j\}=\{1,2\}$, let $H_{i}$ be the graph obtained from $G$ by contracting $Q_{j}$. Then one of $H_{1}$ and $H_{2}$ is planar.

Proof. There is nothing to prove if $G$ itself is planar. So, assume that $G$ is not planar and has a non-contractible circuit. 
I Assume that $G$ has a non-contractible circuit $C$ such that $T \cap E(C)=\emptyset$. Then, without loss of generality, let $Q_{1}$ be the component of $G \backslash T$ such that $E(C) \cap Q_{1}=\emptyset$. So, by Lemma 2.2.3, in the cross cap $\mathcal{P}_{C}, Q_{1}$ contains no vertex of the boundary of the cross cap, and therefore, $H_{1}$ is a planar graph which is embedded in the open disk $\mathcal{P}_{C} \backslash C$.

II By I, we assume that every non-contractible circuit of $G$ must intersect both $Q_{1}$ and $Q_{2}$. Assume that $T \cap E(C)=\left\{e_{1}, e_{2}\right\}$ where $e_{1}=v_{1} v_{2}$, $e_{2}=v_{i} v_{i+1}$. Without considering the cross cap, the graph $G$ in the projective plane can be viewed as a planar graph $G^{*}$ on a closed disk with $C^{*}$ as the boundary of the disk (hence, $v_{\mu}^{\prime}$ and $v_{\mu}^{\prime \prime}$ are viewed as "different" vertices in an embedding $\pi_{C}^{\prime}$, where $v_{1}^{\prime} v_{2}^{\prime}, v_{1}^{\prime \prime} v_{2}^{\prime \prime}, v_{i}^{\prime} v_{i+1}^{\prime}, v_{i}^{\prime \prime} v_{i+1}^{\prime \prime}$, and $e_{3}$ are corresponding to the three edges of $T)$. Let $G_{x}^{*}=G^{*} \backslash\left\{v_{1}^{\prime} v_{2}^{\prime}, v_{1}^{\prime \prime} v_{2}^{\prime \prime}, v_{i}^{\prime} v_{i+1}^{\prime}, v_{i}^{\prime \prime} v_{i+1}^{\prime \prime}, e_{3}\right\}$ and let $Q_{i}^{*}$ be the subgraph of $G_{x}^{*}$ corresponding to $Q_{i}$ of $G(i=1,2)$.

III In the planar graph $G_{x}^{*}$, if there exists a $v_{\mu}^{\prime} v_{\mu}^{\prime \prime}$-path $P$ contained in some $Q_{\mu}^{*}$ for some $\mu$ and $\mu \in\{1,2\}$, then $Q_{i}$ contains a closed non-contractible circuit $v_{\mu} P v_{\mu}\left(v_{\mu}^{\prime}\right.$ and $v_{\mu}^{\prime \prime}$ are identified as $v_{\mu}$ in $G$ ). But, this contradicts the assumption (in II) that no non-contractible circuit is completely contained in one component of $G \backslash T$. So, we assume that both $Q_{1}^{*}$ and $Q_{2}^{*}$ are disconnected in the planar graph $G_{x}^{*}$.

Let those components of $Q_{1}^{*}$ and $Q_{2}^{*}$ in $G_{x}^{*}$ be $Q_{11}^{*}, Q_{12}^{*}\left(\subseteq Q_{1}^{*}\right)$, and $Q_{21}^{*}, Q_{22}^{*}$ $\left(\subseteq Q_{2}^{*}\right)$, respectively, with the segments of $C^{*}$ such that $v_{i+1}^{\prime \prime} \cdots v_{r}^{\prime \prime} v_{1}^{\prime} \subseteq Q_{11}^{*}$, $v_{i+1}^{\prime} \cdots v_{r}^{\prime} v_{1}^{\prime \prime} \subseteq Q_{12}^{*}, v_{2}^{\prime} \cdots v_{i}^{\prime} \subseteq Q_{21}^{*}, v_{2}^{\prime \prime} \cdots v_{i}^{\prime \prime} \subseteq Q_{22}^{*}$ respectively. Without loss of generality, let $e_{3}(\in T)$ be an edge joining $Q_{11}^{*}$ and $Q_{21}^{*}$ ( see the figure). 


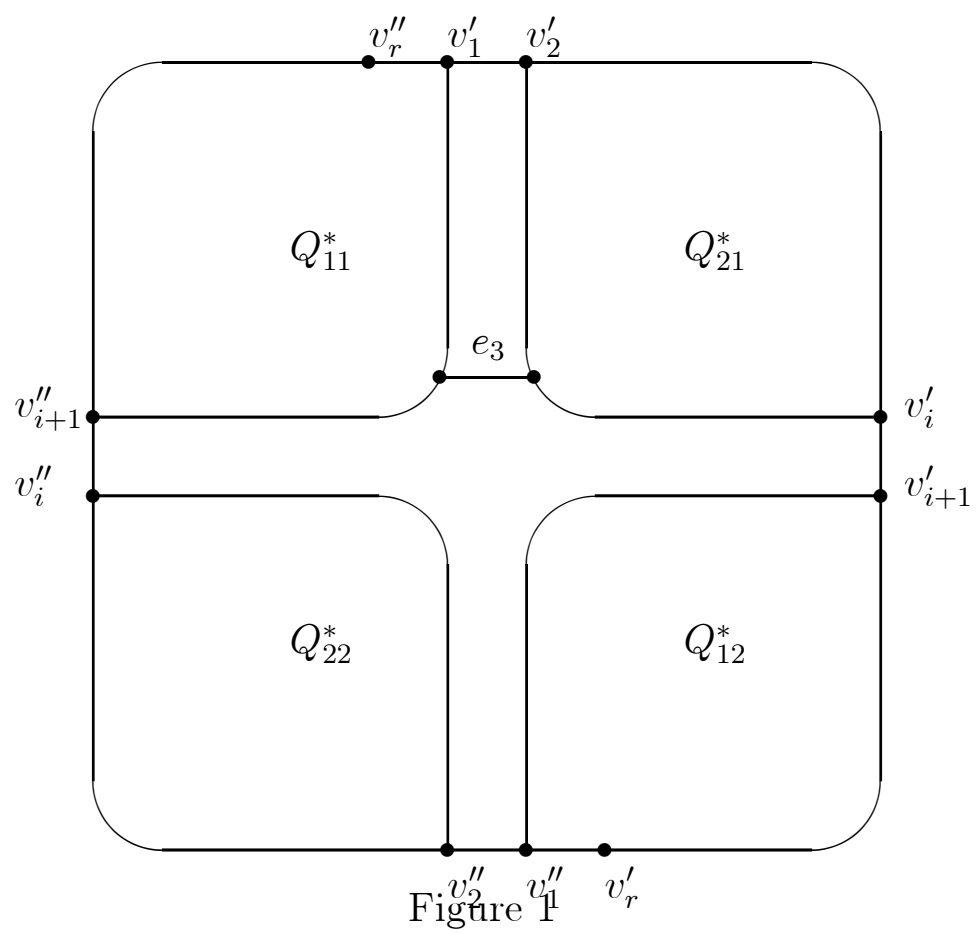

IV Let $\phi$ be a closed non-contractible curve of the projective plane passing through the edges $e_{2}=v_{i}^{\prime \prime} v_{i+1}^{\prime \prime}=v_{i}^{\prime} v_{i+1}^{\prime}$, but no any other point of $G$ since $e_{3}$ is the only edge joining $Q_{11}^{*}$ and $Q_{21}^{*}$ in $G_{x}^{*} \backslash\left\{v_{1} v_{2}, v_{i} v_{i+1}\right\}$ (see Figure 1 ).

Thus, the projective plane $P$ can be represented as a cross cap $\mathcal{P}_{\phi}$ using $\phi$ as the boundary of the cross cap, the boundary $\phi$ cross only one edge $v_{i} v_{i+1}$, but not others. By Lemma 2.2.4, $G$ is a planar graph which contradicts the fact that $G$ is not planar.

A subset $S$ of $V(G)$ is called a separator of $G$ if $G$ has two subgraphs $H_{1}$ and $H_{2}$ such that $G=H_{1} \cup H_{2}$ and $V\left(H_{1}\right) \cap V\left(H_{2}\right)=S$. Denote $S$ by $\left[H_{1}, H_{2}\right]$. 
Lemma 2.2.6 Let $G$ be a 3-connected graph with an embedding $\pi$ in a projective plane. Assume that $G$ has a separator $S$ with $|S|=3, G=G_{1} \cup G_{2}$ and $V\left(G_{1}\right) \cap V\left(G_{2}\right)=S$. Then one of $G_{i}(i=1,2)$ must be a planar graph.

Proof. Let $S=\left\{x_{1}, x_{2}, x_{3}\right\}=\left[G_{1}, G_{2}\right]$. Let $G_{1}^{\prime}$ be a graph obtained from $G_{1}$ by replacing $x_{i}$ with $x_{i}^{\prime}$. Let $G_{2}^{\prime}$ be a graph obtained from $G_{2}$ by replacing $x_{i}$ with $x_{i}^{\prime \prime}$. Let $G^{\prime}$ be a new graph obtained from $G_{1}^{\prime}$ and $G_{2}^{\prime}$ by adding edges $x_{i}^{\prime} x_{i}^{\prime \prime}(i=1,2,3)$. Note that three edges $\left\{e_{1}, e_{2}, e_{3}\right\}$ is a 3 -edge-cut of $G^{\prime}$. By Lemma 2.2.5, one of $G_{1}^{\prime}$ and $G_{2}^{\prime}$ must be a planar. Thus, one of $G_{1}$ and $G_{2}$ must be a planar.

\subsection{Main results}

Theorem 2.3.1 Let $G$ be a 3-connected graph embedded in a projective plane with $\delta \geq 4$. Then every longest circuit of $G$ must have a chord.

Proof. Since the result of this theorem for planar graph was proved in [17], we pay attention only to non planar graphs. Let $C$ be a chordless longest circuit of $G$ that satisfies the hypotheses of the theorem.

A vertex subset $S$ is called a separating 3-vertex-cut with respect to $C$ if $S$ separates $V(G)$ into two parts $V^{\prime}$ and $V^{\prime \prime}$ such that $C$ intersects both $V^{\prime}$ and $V^{\prime \prime}$ and where $|S|=3 . C$ is separable if there is a separating 3-vertexcut with respect to $C$.

I We claim that $C$ is separable. Assume not, that is, $C$ is not separable. 
(i) We claim that every edge of $G$ is incident with two distinct faces. If not, then there is a closed non-contractible curve $\phi$ of the projective plane $P$ that intersects with $G$ at only one vertex of $e$. By Lemma 2.2.4, $G$ is planar. Since we consider only non-planar graphs, every edge of $G$ is incident with two distinct faces. So we can choose an edge $e \in E(G)$ and a face $F$ which is incident with $e$ such that $E(F) \neq E(C)$.

(ii) For the face $F$, there exists an $\{F\}$-Tutte circuit $C_{T}$ of $G$ containing $e$ by Lemma 2.2.2.

(iii) We claim that $V(C) \backslash V\left(C_{T}\right) \neq \emptyset$.

(iii-(a))Case $1 \quad E(F)=E\left(C_{T}\right)$. Then $E\left(C_{T}\right) \neq E(C)$ by (i). If $V(C) \subseteq$ $V\left(C_{T}\right)$, then $V\left(C_{T}\right)=V(C)$ since $\mathrm{C}$ is a longest circuit of $G$. Thus, by (i), all the edges in $E\left(C_{T}\right) \backslash E(C)$ must be the chords of $C$, contradicting the assumption that $C$ is chordless.

(iii-(b)) Case $2 E(F) \neq E\left(C_{T}\right)$. Then $C_{T}$ has some chords by the definition of Tutte-circuit. If $V(C) \subseteq V\left(C_{T}\right)$, then $V\left(C_{T}\right)=V(C)$ since $\mathrm{C}$ is a longest circuit of $G$. Let $E_{1}=E(G[V(C)])$ and $E_{2}=E\left(G\left[V\left(C_{T}\right)\right]\right)$. Here $E_{1}=E_{2}$ since $V(C)=V\left(C_{T}\right)$. This implies that $\left|E_{1} \backslash E(C)\right|$ is the same as the number of chords of $C_{T}$ (which equals $\left|E_{2} \backslash E\left(C_{T}\right)\right|$ ). Note that the Tutte-circuit $C_{T}$ does have chords. This contradicts to the fact that $C$ is chordless.

(iv) We claim that the Tutte circuit $C_{T}$ has a bridge $B$ such that $V(B) \cap$ 
$V(C) \neq \emptyset$. If not, that is, every bridge $B$ of $C_{T}$ contains no vertex of $C$. Then $V(C) \subseteq V\left(C_{T}\right)$. This contradicts (iii).

(v) By (iv), let $B$ be a non-chord bridge of the Tutte circuit $C_{T}$ with

$$
V(B) \cap V(C) \neq \emptyset
$$

Since $C$ is not separable, the 3 -vertex-cut $A(B)$ (the attachments of $B$ ) cannot separate vertices of $V(C)$. Thus,

$$
V(C) \subseteq A(B) \cup V(B)
$$

and

$$
V\left(C_{T}\right) \subseteq G \backslash V(B)
$$

$$
V(C) \cap V\left(C_{T}\right) \subseteq A(B) .
$$

(vi) Let $A(B)=\{x, y, z\}$. Note that the edge $e$ is in both $C$ and $C_{T}$. Let $e=x y$. Here, $x, y \in V(C) \cap V\left(C_{T}\right)$ since $e \in E(C) \cap E\left(C_{T}\right)$. Furthermore, we have that

$$
z \in V(C)
$$

for otherwise, $V(C) \cap V\left(C_{T}\right)=\{x, y\}$ and we have a circuit $x C y C_{T} x$ longer than $C$, a contradiction. 
(vii) We claim that $\left|V\left(C_{T}\right)\right|>3$. If $\left|V\left(C_{T}\right)\right|=3$, then, by (vi), $V\left(C_{T}\right)=$ $A(B)=V\left(C_{T}\right) \cap V(C)$. Since $V(C) \backslash V\left(C_{T}\right) \neq \emptyset$ (by (iii)), $C \neq C_{T}$, and therefore $E\left(C_{T}\right) \backslash E(C) \neq \emptyset$. Hence, each edge of $E\left(C_{T}\right) \backslash E(C)$ is a chord of $C$, a contradiction.

(viii) By (v), $z \in V(C)$ and $C_{T}$ is of length $\geq 4$ by (vii). Therefore, by (iii) either the segment $y C_{T} z$ or the segment $z C_{T} x$ has at least one vertex $v$ that is not in $C$. Say, $v \in z C_{T} x \backslash V(C)$. Since $G$ is 3-connected, there is a path $P$ joining $z C_{T} x-\{x, z\}$ and $y C_{T} z-\{z\}$ in $G \backslash\{x, z\}$. Choose $P$ as short as possible. Let $P=u^{\prime} \cdots u^{\prime \prime}$ with $u^{\prime} \in z C_{T} x-\{x, z\}$ and $u^{\prime \prime} \in y C_{T} z-\{z\}$. If $[V(P) \backslash\{y\}] \cap V(B)=\emptyset$, then $u^{\prime} C_{T} x C y C_{T} u^{\prime \prime} P u^{\prime}$ is a circuit longer than $C$. So $[V(P) \backslash\{y\}] \cap V(B) \neq \emptyset$, then $u^{\prime} \in A(B)$ or $u^{\prime \prime} \in A(B)$ if $u^{\prime \prime} \neq y$ (since $P$ is shortest). But this contradicts $B$ being a 3 -attachment bridge with $A(B)=\{x, y, z\}$.

II By I, $C$ is separable. Let $V^{*}$ be a separable 3-vertex-cut with respect to $C$ such that $V^{*}$ separates $G$ into $V^{\prime}$ and $V^{\prime \prime}$. By Lemma 2.2.5 one of $G\left[V^{\prime} \cup V^{*}\right], G\left[V^{\prime \prime} \cup V^{*}\right]$ must be planar. Without loss of generality, assume that $G\left[V^{\prime \prime} \cup V^{*}\right]$ is a planar. We choose $V^{*}$ such that $V^{\prime \prime}$ is as small as possible.

Since $C$ must pass through two vertices of $V^{*}$ to enter $V^{\prime \prime}$ from $V^{\prime}$, the parts of $C$ in $G\left[V^{\prime} \cup V^{*}\right]$ and $G\left[V^{\prime \prime} \cup V^{*}\right]$ are paths. Let $C \cap G\left[V^{\prime} \cup V^{*}\right]=$ $P^{\prime}=x \cdots y$, and $C \cap G\left[V^{\prime \prime} \cup V^{*}\right]=P^{\prime \prime}=y \cdots x$. Obviously, $x, y \in V^{*}$. Let $V^{*}=\{x, y, z\}$. We construct a new graph $G^{*}$ according to the following two cases: 
(a) If $z \notin V\left(P^{\prime}\right)$, let $w$ be a new vertex not in $C$, add the vertex $w$ to $V\left(G\left[V^{\prime \prime} \cup V^{*}\right]\right)$ and add three new edges $\{w x, w y, w z\}$ to $E\left(G\left[V^{\prime \prime} \cup V^{*}\right]\right)$. Denote the new graph $G^{*}$.

(b) If $z \in V\left(P^{\prime}\right)$, let $w=z$ and

$$
G^{*}=G\left[V^{\prime \prime} \cup V^{*} \cup\{w\}\right] \cup\{w x, w y\}
$$

Obviously, $G^{*}$ is still planar.

Let $C^{*}$ be the graph obtained from $P^{\prime \prime}$ by adding the vertex $w$ and edges $w x, w y$. Here, $C^{*}$ is a longest circuit of $G^{*}$ containing $w x$ and $w y$.

Let $F_{1}, F_{2}$ be two faces of $G$ containing $w x$. There is an $\left\{F_{1}, F_{2}\right\}$-Tutte circuit $C^{o}$ of $G^{*}$ that contains $w x$ and $w y$ (by Lemma 3.2).

(i) If $\left|V(C) \cap V^{\prime \prime}\right|=1$, then let $\{v\}=V(C) \cap V^{\prime \prime}$ and $P^{\prime \prime}$ must be one of $\{x v y, x v z y, x z v y\}$. Without loss of generality, we consider the case $\{x v y, x v z y\}$. let $P^{\prime \prime}=w_{1} \cdots w_{t}$ where $w_{1}=x, w_{t}=y, w_{2}=v$ if $t=3$ and $w_{3}=z$ if $t=4$, (note the case $\{x v y, x z v y\}$ is similar to the case $\{x v y, x v z y\}$ ).

Since the minimum degree of $G$ is at least four, there are at least two edges adjacent to $v$ not contained in $C$. Let $v u$ be an edge not contained in $C$ such that $z \neq u$. Clearly $u \in V^{\prime \prime}$. Let $B_{C}$ be the $C$ - bridge of $G$ containing $u$. If $w_{1}$ or $w_{3}$ is in the attachment of $B_{C}$, then $P^{*}=w_{1} P_{w_{1}} u v P^{\prime \prime} w_{t}$ (where $P_{w_{1}}$ is the longest path in $B_{C}$ connecting $w_{1}$ and $u$ ) or $P^{*}=x v u P_{w_{3}} w_{3} P^{\prime \prime} w_{t}$ (where $P_{w_{3}}$ is the longest path in $B_{C}$ connecting $w_{3}$ and $u$ ) would be longer than $P^{\prime \prime}$ in $G^{*}$, C would not be a longest circuit. Hence, neither $w_{1}$ nor $w_{3}$ is in attachment set of $B_{C}$. Note that every path joining $u$ and a vertex in $V(C)$ must pass through $\left\{w_{4}, v\right\}$ if $t=4$ or $\{z, v\}$ if $t=3$, which contradicts $G$ being 3-connected. Hence, $\left|V(C) \cap V^{\prime \prime}\right| \geq 2$. 
(ii) Since $V^{\prime \prime}$ is minimum and $\left|V(C) \cap V^{\prime \prime}\right| \geq 2$, so $\left|N(v) \cap V^{\prime \prime}\right| \geq 2$ for any $v \in V^{*}$. Let $x x_{i}$ be the edge of $G^{*}$ lying on the boundary of $F_{i}$ and $x x_{i} \neq x w$ for $i=1,2$. Obviously, $x x_{1}, x x_{2} \in E(G)$. Since $C^{o}$ is an $\left\{F_{1}, F_{2}\right\}-$ Tutte circuit and because $G^{*}$ is 3 -connected, $x x_{i}$ either lies on $C^{o}$ or is chord of $C^{o}$. Hence, $\left\{x, x_{1}, x_{2}\right\} \subseteq V\left(C^{o}\right)$ and one of $\left\{x x_{1}, x x_{2}\right\}$ must be a chord of $C^{o}$ since $x w \in E\left(C^{o}\right)$.

(iii) We claim that $V\left(C^{o}\right) \backslash\{x, y, z, w\} \neq \emptyset$. Assume that $V\left(C^{o}\right) \backslash\{x, y, z, w\}$ $=\emptyset$, then by (ii), $\left\{x_{1}, x_{2}\right\}=\{y, z\}$ and since $V(C) \cap V^{\prime \prime} \neq \emptyset, x y$ is a chord of $C$. This contradicts $C$ being chordless. Hence, $V\left(C^{o}\right) \backslash\{x, y, z, w\} \neq \emptyset$.

(iv) We claim that each nonchord bridge $B$ of $C^{o}$ must be contained in some bridge of $C^{*}$. Suppose that $V(B) \cap V\left(C^{*}\right) \neq \emptyset$ for some bridge $B$ of $C^{o}$.

(iv- $\alpha$ ) Case $1 \quad w \notin A(B)$ or $w=z$. If $w \notin A(B)$, then $z \notin V(B)$ since $C^{o}$ contains edges $w x$ and $w y$. If $w=z$, then $w=z \notin V(B)$. Hence $x, y, z \notin V(B)$ and $V^{\prime}$ adjacent only with $\{x, y, z\}$ in $G$ will imply that $A(B)$ is a vertex-cut that separates $G$ into $V(B)$ and $V(G) \backslash[A(B) \cup V(B)]$. Since $V\left(C^{o}\right) \backslash\{x, y, z, w\} \neq \emptyset$ ( by (iii)), $V(B)$ would be a proper subset of $V^{\prime \prime}$. Howerer, $V(B)$ intersects with $C$, which contradicts to the choice of $V^{*}$ with $V^{\prime \prime}$ minimum.

(iv- $\beta$ ) Case $2 w \in A(B)$ and $w \neq z$. Since $\{x, y, w\} \subseteq V\left(C^{o}\right)$ and $w \in A(B)$, we must have that $z \in V(B)$ and $w z \in[V(B), A(B)]$. Since $C^{o}$ is an $\left\{F_{1}, F_{2}\right\}$-Tutte circuit and $w z \in E\left(F_{1} \cup F_{2}\right)$. B is a 2-attachment bridge. Since $d(z) \geq 3$ in $G^{*}, V(B) \backslash\{z\} \neq \emptyset$. Let $A(B)=\{w, u\}$. Then $U^{*}=$ 
$\{z, u\}$ is a vertex-cut of $G^{*}$ because $d(w)=3$ and hence $w \in A(B)$. $U^{*}$ would separate $G^{*}$ into $U^{\prime \prime}=V(B) \backslash\{z\}$ and $U^{\prime}=\left[V\left(G^{*}\right) \backslash[V(B) \cup A(B))\right] \cup\{w\}$. Since $\{x, y, z, w\} \subseteq U^{\prime} \cup U^{*}, V^{\prime}$ only adjacent with $V^{*}=\{x, y, z\}$ would imply that $V^{\prime}$ and $U^{\prime \prime}$ are disconnected in $G \backslash U^{*}$. Hence, $U^{*}$ is a 2-vertex-cut separating $G$ into $U^{\prime \prime}$ and $V^{\prime} \cup U^{\prime} \backslash\{w\}$, which contradicts $C$ being 3-connected . Now we conclude our claim in all cases.

(v) By (iv), $V\left(C^{*}\right) \subseteq V\left(C^{o}\right)$ since each bridge of $C^{o}$ is contained in some bridge of $C^{*}$. Moreover, $V\left(C^{o}\right)=V\left(C^{*}\right)$ because $C^{*}$ is a longest circuit of $G^{*}$ containing $w x$ and $w y$. By (ii), $\left\{x, x_{1}, x_{2}\right\} \subseteq V\left(C^{o}\right)=V\left(C^{*}\right)$ and one of $\left\{x x_{1}, x x_{2}\right\}$ is a chord of $C^{*}$, which is also a chord of $C$ in $G$. This contradicts $C$ being chordless. This completes the proof. 


\section{Chapter 3}

\section{Multiple Chords of Longest Circuits in 3-Connected Graphs}

\subsection{Introduction}

We have proved in Chapter 2 that every longest circuit of 3-connected graph embedded in projective plane has a chord. A graph is said to be cubic if every vertex has of degree 3 . In this chapter, we prove that every longest circuit of a 3-connected cubic graph has at least two chords. Furthermore, if the graph is also planar, then every longest circuit has at least three chords.

In 1997, Carsten Thomassen [14] proved that every longest circuit in a 3-connected cubic graph has a chord. With a similar method and more detail,we prove that the same circuit has at least two chords. If this graph is planar, then has at least 3 chords.

A underlying graph of $G$ is the graph induced from $G$ by subsititing each path $P=x_{1} x_{2} \ldots x_{s}$ of $G$ by an edge $x_{1} x_{s}$ where $d_{G}\left(x_{i}\right)=2(1 \leq i \leq s)$. 
Multiple Chords of Longest Circuits in 3-Connected Graphs

\subsection{Multiple chords of longest circuits in cu- bic graphs}

Lemma 3.2.1 (Fleischner and Stiebitz [3]) Let $n$ be a positive integer, and let $G$ be a 4-regular graph on $3 n$ vertices. Assume that $G$ has a decomposition into a Hamilton circuit and $n$ pairwise vertex disjoint triangles. Then $\chi(G)=$ 3.

Lemma 3.2.2 Let $C$ be a Hamilton circuit of a graph $G$, and $V(G)=V(C)$ have a partition $\{A, B\}$ such that

(1) $A$ is an independent set of $C$ ( may not be independent in $G$ ), and

(2) $G \backslash A$ has $|A|$ components.

Then, for each $v \in A$, every Hamilton path of $G$ starting at $v$ must end at a vertex of $B$.

Proof Let $P=v \cdots x$ be a Hamilton path of $G$ starting at $v \in A$. If $x \notin B$, then $G \backslash A \supseteq P \backslash A$ has at most $|A|-1$ components since both endvertices $x$ and $v$ of $P$ are in $A$. This contradicts the condition (2).

Lemma 3.2.3 (Thomason, [14])

Let $G$ be a graph described in Lemma 3.2.2 such that $d(w)$ is odd for every $w \in B$. Let $v \in A$ and $e$ be an edge of $C$ incident with $v$. Then the number of Hamilton circuits of $G$ containing the edge e must be even.

Proof Let $\mathcal{P}$ be the set of all Hamilton paths in $G$ starting at $v$ and $e$. Construct an auxiliary graph $H$ as follows: $V(H)=\mathcal{P}$ and for each pair 
$P_{1}=x_{1} \cdots x_{n}, P_{2}=y_{1} \cdots y_{n}\left(\right.$ where $x_{1}=y_{1}=v$ and $\left.x_{1} x_{2}=y_{1} y_{2}=e\right)$ of $\mathcal{P}, P_{1}$ and $P_{2}$ are adjacent in $H$ if and only if $x_{n}$ is adjacent to a vertex $x_{t}$ $(t \geq 2)$ such that $P_{2}=x_{1} P_{1} x_{t} x_{n} \overline{P_{1}} x_{t+1}$.

For each $P=u_{1} \cdots u_{n} \in \mathcal{P}$, it is obvious that

(1) the vertex $P$ in $H$ must be of degree $d_{G}\left(u_{n}\right)-1$ if $u_{1} u_{n} \notin E(G)$.

(2) the vertex $P$ in $H$ must be of degree $d_{G}\left(u_{n}\right)-2$ if $u_{1} u_{n} \in E(G)$.

By Lemma 3.2.2, $u_{n} \in B$. Therefore, $P=u_{1} \cdots u_{n}$ must be of even degree if $u_{1} u_{n} \notin E(G)$, and must be of odd degree if $u_{1} u_{n} \in E(G)$. Since the number of odd degree vertices of $H$ must be even, the number of members $P \in \mathcal{P}$ with $u_{1} u_{n} \in E(G)$ is even.

Corollary 3.2.4 Let $C$ be a Hamilton circuit in the graph $G$. If $C$ has a vertex set $A$ such that

(1) $A$ is independent in $C$ ( may not be independent in $G$ ) and

(2) every vertex not in $A$ is joined to a vertex in $A$ by an edge in $E(G) \backslash$ $E(C)$.

Then, for each vertex $v \in A$ and each edge $e \in E(C)$ incident with $v$, the number of Hamilton circuits of $G$ containing the edge e must be more than one.

Proof Let $G^{\prime}$ be a subgraph of $G$ which consists of all vertices of $G$, all edges of $C$ and precisely one edge of $E(G) \backslash E(C)$ joining $x$ and $A$ for each vertex $x$ not in $A$. Then, each vertex of $G^{\prime} \backslash A$ has degree 3 , so, by Lemma 3.2.3, $G$ has a second Hamilton circuit containing the edge $e$. 
Lemma 3.2.5 Let $C$ and $C^{\prime}$ be two different Hamilton circuits of a simple graph $G$ of order $\geq 5$. Let $C=v_{1} v_{2} \cdots v_{r} v_{1}(r \geq 5)$ in clockwise order. Then

(1) $\left|E\left(C^{\prime}\right) \backslash E(C)\right| \geq 2$ and

(2) if $\left|E\left(C^{\prime}\right) \backslash E(C)\right|=2$, then $E\left(C^{\prime}\right) \backslash E(C)$ must be a pair of chords $\left\{v_{a} v_{b}, v_{a-1} v_{b-1}\right\}$ of $C$.

Proof. Since $C \neq C^{\prime},|E(C)|=\left|E\left(C^{\prime}\right)\right|$ and $G$ is simple, we have that $\left|E\left(C^{\prime}\right) \backslash E(C)\right|=\left|E(C) \backslash E\left(C^{\prime}\right)\right|$. And $C \triangle C^{\prime}$ is an even subgraph of $G$ with $\left|E\left(C^{\prime}\right) \backslash E(C)\right|=\frac{1}{2}\left|E\left(C \triangle C^{\prime}\right)\right| \geq \frac{4}{2}=2$. (If $\left|E\left(C \triangle C^{\prime}\right)\right|=2$, then $\left|E\left(C^{\prime}\right) \backslash E(C)\right|=\left|E(C) \backslash E\left(C^{\prime}\right)\right|=1$ which is impossible since $G$ is simple ). This proves (1).

If $\left|E\left(C^{\prime}\right) \backslash E(C)\right|=2$, then $\left|E(C) \backslash E\left(C^{\prime}\right)\right|=2$.

Let $E\left(C^{\prime}\right) \backslash E(C)=\left\{v_{\alpha} v_{\beta}, v_{\gamma} v_{\delta}\right\}$. Note that $C^{\prime} \backslash\left\{v_{\alpha} v_{\beta}, v_{\gamma} v_{\delta}\right\}$ is a set of 2 paths $Q_{1}, Q_{2}$ with end vertices in $\left\{v_{\alpha}, v_{\beta}, v_{\gamma}, v_{\delta}\right\}$. Since $Q_{1}$ and $Q_{2}$ are also segments of $C$, and $\left|E(C) \backslash\left\{E\left(Q_{1}\right) \cup E\left(Q_{2}\right)\right\}\right|=2$ ( note that $\left|E\left(C^{\prime}\right)\right|=|E(C)|$ ), we must have that $v_{\gamma}=v_{\alpha \pm 1}$ and $v_{\delta}=v_{\beta \pm 1}$. This proves (2).

Theorem 3.2.6 Let $G$ be a 3-connected cubic graph. Then every longest circuit of $G$ must have at least two chords.

Proof Let $C$ be a longest circuit of $G$ which has only one chord. Since $G$ is 3-connected and cubic, it is easy to check the Theorem is true for $|E(C)| \leq 5$. So we assume that $|E(C)| \geq 6$. Let $e=x y$ be the only chord that $C$ has. Let $x^{\prime}, x^{\prime \prime}$ be the neighbor of $x$ in $C$ and $y^{\prime}, y^{\prime \prime}$ be the neighbor of $y$ in $C$. 
Delete $e$ from $G$. Let the resulting underlying graph be $G^{\prime}=\overline{G-e}$ and $C^{\prime}$ be the Hamilton circuit of $G^{\prime}$ corresponding to the $C$ of $G$.

For each component $B$ of $G \backslash V\left(C^{\prime}\right)$, we select three vertices $x(B), y(B)$, $z(B)$ of $C^{\prime}$ which are joined to the vertices of $B$. This is possible since $G$ is 3-connected. Since $G$ is cubic, $\{x(B), y(B), z(B)\} \cap\left\{x\left(B^{\prime}\right), y\left(B^{\prime}\right), z\left(B^{\prime}\right)\right\}=\emptyset$ if $B \neq B^{\prime}$.

We construct a new graph $G^{\prime \prime}$ which consists of $C^{\prime}$ and all triangles of the form $x(B) y(B) z(B) x(B)$. By Lemma 3.2.1, $G^{\prime \prime}$ is 3-colorable.

Let $c: V\left(G^{\prime \prime}\right) \mapsto\{1,2,3\}$. Each $c^{-1}(i)(i=1,2,3)$ is a independent set of $C^{\prime}$. Without loss of generality, let $c\left(x^{\prime}\right)=1$. We construct another new graph $G^{\prime \prime \prime}$ from $G^{\prime}$ by contracting each component $B$ of $G^{\prime} \backslash V\left(C^{\prime}\right)$ into a vertex $x(B) \in A(B) \cap c^{-1}(1)$. So $G^{\prime \prime \prime}$ has another Hamilton circuit $C^{\prime \prime}$ containing $x^{\prime} x^{\prime \prime}$ different from $C^{\prime}$ by the Corollary 3.2.4 $\left(x^{\prime}\right.$ is in the independent set $c^{-1}(1)$ of $\left.C\right)$.

Note that $\left|E\left(C^{\prime \prime}\right) \backslash E\left(C^{\prime}\right)\right| \geq 2$ by Lemma 3.2.5. Though $C^{\prime \prime}$ may miss the edge $y^{\prime} y^{\prime \prime}$, but it must use at least 2 chords of $C^{\prime}$ in $G^{\prime \prime \prime}$. Back to $G$, let $C^{*}$ be the circuit corresponding to $C^{\prime \prime}$. In the circuit $C^{*}$, the vertex $y$ may not be in $C^{*}$ if $y^{\prime} y^{\prime \prime}$ is not in $C^{\prime \prime}$. But those edges in $E\left(C^{\prime \prime}\right) \backslash E\left(C^{\prime}\right)$ must belong to some triangles of form $\{x(B), y(B), z(B)\}$ where $B$ is one of the components of $G \backslash V\left(C^{\prime}\right)$. So they must bring at least two new vertices into $C^{*}$ which are in at least two different components of $G \backslash V\left(C^{\prime}\right)$. So, $C^{*}$ is longer than $C$. This contradicts the assumption that $C$ is a longest circuit.

Theorem 3.2.7 Every longest circuit of a 3-connected cubic planar graph 
has at least 3 chords.

Proof. Since $G$ is 3-connected and cubic, it is easy to check that the theorem is true for $|E(C)| \leq 5$, so we assume that $|E(C)| \geq 6$. Let $C=$ $v_{1} \cdots v_{r} v_{1}(r \geq 6)$ be a longest circuit of a 3 -connected plane graph $G$ in clockwise order. By Theorem 3.2.6, we assume that $C$ has precisely 2 chords $v_{a} v_{b}, v_{c} v_{d}$ (where $1 \leq a<b, c<d$ ).

Construct a graph $H$ from $G$ by

(1) replacing each non-trivial bridge $B$ with a triangle $v_{p} v_{q} v_{r} v_{p}$ with $\left\{v_{p}, v_{q}, v_{r}\right\} \subseteq A(B)$ (where $A(B)$ is the attachment of the bridge $B$ ). This is possible since $G$ is 3 -connected. Since $G$ is cubic, $\left\{v_{p}, v_{q}, v_{r}\right\} \cap\left\{v_{p^{\prime}}, v_{q^{\prime}}, v_{r^{\prime}}\right\}=$ $\emptyset$ if $B \neq B^{\prime}$ ( where $\left\{v_{p^{\prime}}, v_{q^{\prime}}, v_{r^{\prime}}\right\} \subseteq A\left(B^{\prime}\right)$ );

(2) replacing the chords $v_{a} v_{b}, v_{c} v_{d}$ with a triangle $v_{a} v_{b} v_{c} v_{a}$.

Now the underlying graph $\bar{H}$ is vertex-3-colorable by Lemma 3.2.1.

Let $c: V(H) \backslash\left\{v_{d}\right\}=V(\bar{H}) \mapsto\{1,2,3\}$.

Without loss of generality, let $\left\{c\left(v_{d+1}\right), c\left(v_{d-1}\right)\right\}=\{1,2\}$ (note that they are adjacent in $\bar{H})$. Since $c\left(v_{a}\right) \neq c\left(v_{c}\right)$, without loss of generality, let $1 \in$ $\left\{c\left(v_{a}\right), c\left(v_{c}\right)\right\} \cap\left\{c\left(v_{d+1}\right), c\left(v_{d-1}\right)\right\}$.

Let $H$ be the underlying graph of the graph obtained from $G \backslash v_{c} v_{d}$ by contracting each bridge $B$ of $C$ in $G$ other than $v_{c} v_{d}$ into a vertex of $c^{-1}(1) \cap$ $A(B)$. By Corollary 3.2.4, $H$ contains a Hamilton circuit $C^{\prime}$ containing the edge $v_{d-1} v_{d+1}$ and is different from $C$. Note that the edge $v_{c-1} v_{c+1}$ of $C$ may not be in $C^{\prime}$ and $C^{\prime}$ must contain at least two chords of $C$ in $H$. We consider the following four cases:

Case $1 \quad v_{c-1} v_{c+1} \notin E\left(C^{\prime}\right), v_{a} v_{b} \in E\left(C^{\prime}\right)$. 


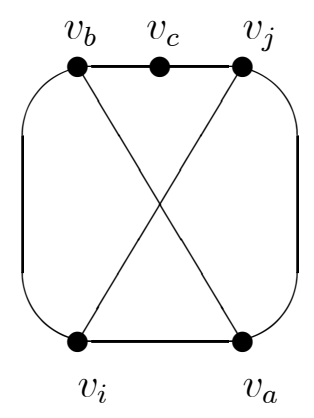

(Figure 2)

Case $2 v_{c-1} v_{c+1} \notin E\left(C^{\prime}\right), v_{a} v_{b} \notin E\left(C^{\prime}\right)$.

Case $3 \quad v_{c-1} v_{c+1} \in E\left(C^{\prime}\right), v_{a} v_{b} \in E\left(C^{\prime}\right)$.

Case $4 v_{c-1} v_{c+1} \in E\left(C^{\prime}\right), v_{a} v_{b} \notin E\left(C^{\prime}\right)$.

Let $p=\left|E\left(C^{\prime}\right) \backslash\left[E(C) \cup\left\{v_{a} v_{b}\right\}\right]\right|$ in $H$. Assume that $p \geq 2$ in Case 1 and Case 2, or $p \geq 1$ in Case 3 and Case 4. Note that each edge of $E\left(C^{\prime}\right) \backslash\left[E(C) \cup\left\{v_{a} v_{b}\right\}\right]$ joins a pair of vertices of $A(B)$ for some non-trivial bridge $B$ of $C$ in $G$. Thus, the circuit in $G$ corresding to $C^{\prime}$ is longer than $C$. This contradicts that $C$ is longest. So, we have that $p=1$ for Case 1 and Case 2, $p=0$ for Case 3 and Case 4. By Lemma 3.2.5, $\left|E\left(C^{\prime}\right) \backslash E(C)\right| \geq 2$, we have that Case 2, Case 3 and Case 4 cannot happen. The only remaining case is Case 1 that $v_{c-1} v_{c+1} \notin E\left(C^{\prime}\right), v_{a} v_{b} \in E\left(C^{\prime}\right)$ and $p=1$. Let $v_{i} v_{j} \in$ $E\left(C^{\prime}\right) \backslash\left[E(C) \cup\left\{v_{a} v_{b}\right\}\right]$ (note that $p=1$ here) where $v_{i}, v_{j} \in A\left(B_{1}\right)$ for some nontrivial bridge $B_{1}$ of $C$ in $G$ and $v_{i}$ is a adjacent to $v_{a}, v_{j}$ is adjacent to $v_{b}$ along the circuit $C$ in $H$ by Lemma 3.2.5(ii). Note that $v_{c-1} v_{c+1} \in E\left(C^{\prime}\right)$ in 
the Case $1, v_{c}$ must be between either $v_{a}$ and $v_{i}$, or $v_{b}$ and $v_{j}$. Without loss of generality, let $i=a+1, c=b+1, j=b+2$. (Figure 2).

With similar arguments, consider the Hamilton circuit $C^{\prime \prime}$ of $H$ containing the edge $v_{c-1} v_{c+1}\left(=v_{b} v_{j}\right)$ (note that one of vertex in $\left\{v_{c-1}, v_{c+1}\right\}$ is in the independent set $c^{-1}(1)$ of $\left.C\right)$. And $v_{d-1} v_{d+1}\left(=v_{a-2} v_{a}\right) \notin C^{\prime \prime}, v_{a} v_{b} \in C^{\prime \prime}$, $\left|E\left(C^{\prime \prime}\right) \backslash\left[E(C) \cup\left\{v_{a} v_{b}\right\}\right]\right|=1$. Let $v_{r} v_{q} \in E\left(C^{\prime \prime}\right) \backslash\left[E(C) \cup\left\{v_{a} v_{b}\right\}\right]$, without loss of generality, let $v_{r}$ adjacent to $v_{b}$ and $v_{q}$ adjacent to $v_{a}$ by Lemma 3.2.5. Since $c=b+1$ and $C^{\prime \prime}$ pass through edge $v_{c-1} v_{c+1}$, so $r=b-1, q=a-1$ (see Figure 3). Now $C \cup C^{\prime} \cup C^{\prime \prime}$ contains a subdivition of $K_{3,3}$ which is impossible since $G$ is a planar.

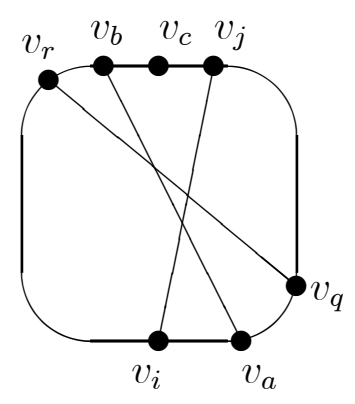

(Figure 3) 


\section{Chapter 4}

\section{Chords of Graphs Embedded in the Torus and Klein Bottle}

\subsection{Introduction and Terminology}

A torus is a surface in three dimensional real space obtained by rotating a circle about a line which lies in the plane of the circle but does not intersect the circle. A torus may be thought of as the sphere with one handle.

A Klein bottle is a one-sided surface which can be made from a cylindrical surface with the two ends of this cylinder to be identified.

In this chapter,we shall prove the following theorem:

Theorem 4.1.1 (The Main Theorem) Every longest circuit of a 4-connected 
graph embedded in the torus or the Klein bottle has a chord.

The method that we shall use in the proof of Theorem 4.1.1 is very different from those in papers ([14],[15]) and [17]. Methods used in those papers are based on connectivity, enumeration, Hamilton circuits and vertex coloring. Here, we use Euler contribution, charge-discharge method.

Let $u, v \in V(G)$. The vertex $u$ is a neighbor of $v$ if $u v \in E(G)$. The set of all neighbors of $v$ is denoted by $N(v)$ and the set of edges incident with the vertex $v$ is denoted by $E(v)$. The degree of a vertex $v$, denoted by $d_{G}(v)$ (or simply, $d(v)$, if there is no confusion), is the number of neighbors of $v$. For a subgraph $P$ of $G, d_{P}(v)=|N(v) \cap V(P)|$.

The length of the boundary of a face $f$ (or, simply, the length of $f$, the degree of $f$ ) of a graph $G$ is denoted by $d_{G}(f)$.

Let $C=v_{1} v_{2} \ldots v_{i} v_{i+1} \ldots v_{m} v_{1}$ be a longest circuit of a 4-connected graph $G$. Let $v_{i}, v_{j} \in V(C)$ with $i<j$, the segment $v_{i} v_{i+1} \ldots v_{j-1} v_{j}$ of $C$ is denoted by $v_{i} C v_{j}$, the segment $v_{j} v_{j-1} \ldots v_{i+1} v_{i}$ of $C$ is denoted by $v_{j} \bar{C} v_{i}$.

Let $G$ be embedded in a surface $S$. If each face of $G$ is isomorphic to an open disk, then this embedding is called an open 2-cell embedding. Note that every graph has an open 2-cell embedding in some surface and all embeddings we considered in this chapter are open 2-cell embeddings.

For the sake of convenience in the later discussion, if $e=x y$ and $f^{\prime}, f^{\prime \prime}$ are faces on the two sides of the edge $e$, we say that the edge $e$ is associated 
with sequence $\left\{x, y, f^{\prime}, f^{\prime \prime}\right\}$. (It is possible that $f^{\prime}$ may be the same as $f^{\prime \prime}$.)

\subsection{Euler Contribution}

Let a graph $H$ be embedded in a surface. For a vertex $v \in V(H)$, let $\left\{e_{1}, \ldots, e_{d(v)}\right\}=E(v)$ where $e_{i}, e_{i+1}(\bmod d(v))$ are on the boundary of a face. An angle $\alpha$ (at $v)$ of $G$ is a pair of edges $\left\{e_{i}, e_{i+1}\right\}(\bmod d(v))$.

Denote the set of all angles of $H$ by $\Lambda$. For an angle $\alpha \in \Lambda$ at a vertex $v$ and at a corner of a face $f$, denote the vertex $v$ by $v_{\alpha}$ and face $f$ by $f_{\alpha}$. Note that there are $d(v)$ angles at vertex $v$ and there are $d(f)$ angles at the corners of a face $f$ and each edge appears in four angles and each angle consists of two edges.

$$
\begin{gathered}
|V(H)|=\sum_{\alpha \in \Lambda(G)} \frac{1}{d\left(v_{\alpha}\right)}, \\
|E(H)|=\sum_{\alpha \in \Lambda(G)} \frac{1}{2}, \\
|F(H)|=\sum_{\alpha \in \Lambda(G)} \frac{1}{d\left(f_{\alpha}\right)} .
\end{gathered}
$$

From Eular's formula for the torus and the Klein bottle

$$
|V(H)|-|E(H)|+|F(H)|=0,
$$

we have Lebesgue's formula $([6])$

$$
\sum_{\alpha \in \Lambda(H)}\left(\frac{1}{d\left(v_{\alpha}\right)}+\frac{1}{d\left(f_{\alpha}\right)}-\frac{1}{2}\right)=0 .
$$


For each angle $\alpha$, the term

$$
\Phi(\alpha)=\frac{1}{d\left(v_{\alpha}\right)}+\frac{1}{d\left(f_{\alpha}\right)}-\frac{1}{2}
$$

of (1) is called the Euler contribution of the angle $\alpha$.

For an edge $e=v_{1} v_{2}$, let $f_{1}, f_{2}$ be the faces incident with $e$. Note that $e$ appears in four angles and each angle consists of two edges. When one sums a half of the Euler contribution of all angles containing $e$, one obtains the Euler contribution of the edge $e$

$$
\Phi(e)=\frac{1}{d\left(v_{1}\right)}+\frac{1}{d\left(v_{2}\right)}+\frac{1}{d\left(f_{1}\right)}+\frac{1}{d\left(f_{2}\right)}-1 .
$$

According to Lebesgue's formula (1), we have the total Euler contributions of all angles and all edges

$$
\sum_{\alpha \in \Lambda(H)} \Phi(\alpha)=\sum_{e \in E(G)} \Phi(e)=0 .
$$

\subsection{Lemmas and Definitions}

The application of Euler contribution and the search for edges with positive Euler contributions will lead our attention to the local structure of some adjacent pairs of small faces. Though the global embedding in the torus or the Klein bottle is different from that in the sphere, the local structure of a subgraph embedded in an open disk neighborhood of the torus or the Klein bottle may appear very similar to the planar graph. In order to avoid any possible misuse of properties that are for the sphere but not for the torus or the Klein bottle, we need two lemmas (Lemma 4.3.2 and Lemma 4.3.3.) which describe some local properties of faces and their boundaries in the 
torus and the Klein bottle. Lemma 4.3.2 (together with some claims, such as claim (5) in the proof) enables us to work on small faces locally without worrying about any complicated structure around the boundaries (very much like in the sphere).

A remark. Note that the embedding we are talking about here is an open 2-cell embedding. That is, each face is isomorphic to an open disk. However, one should not confuse it with a closed 2-cell embedding, in which the closure of every face is isomorphic to a closed disk. Therefore, we cannot assume that the boundary of any face is a circuit, since it is possible that it might pass twice through some edge.

Definition 4.3.1 Let $H$ be a graph embedded in a surface $S$. A face $f$ of $G$ is good if the boundary of $f$ is a circuit. A face $f$ is bad if otherwise.

Lemma 4.3.2 Let $H$ be a connected triangle-free graph with $\delta(H) \geq 2$ which has an open 2-cell embedding in a surface S. Let $f$ be a face of $G$ in $S$. If $f$ is a bad face of $G$, then the length of $f$ must be at least 10 if the surface $S$ is orientable, and is at least 8 if $S$ is non-orientable.

Proof. Let $\mathrm{C}$ be the boundary of a bad face $f$ which closed walk orientation $C=v_{1} \ldots v_{r} v_{1}$. Since $f$ is bad, $C$ is not a circuit. Hence, some edge $e$ is passed twice in $C$.

Case 1. The edge $e$ is passed twice in opposite directions. Assume that $e=x y$ is $v_{1} v_{2}=x y$ and $v_{\mu-1} v_{\mu}=y x$ in the closed walk $C$. Since $\delta(H) \geq 2$, the graph induced by the closed subwalk $v_{2} \ldots v_{\mu-1}$ contains a circuit $Q_{1}$ of length at most $\mu-3$. Furthermore, $Q_{1}$ is of length at least 4 since the graph 
$H$ is triangle-free. Symmetrically, the graph induced by the closed subwalk $v_{\mu} \ldots v_{r} v_{1}$ contains a circuit $Q_{2}$ of length at most $r-\mu+1$, which is also at least 4 . So, it is obvious that $r \geq 10$.

Case 2. The edge $e$ is passed twice in the same direction. (Note that this case occurs only in non-orientable surfaces.) Assume that $e=x y$ is $v_{1} v_{2}=x y$ and $v_{\mu-1} v_{\mu}=x y$ in the closed walk $C$. Since $\delta(H) \geq 2$, the graph induced by the closed subwalk $v_{2} \ldots v_{\mu}$ contains a circuit $Q_{3}$ of length at most $\mu-2$. Furthermore, the circuit $Q_{3}$ is of length is at least 4 since the graph is triangle-free. Symmetrically, the graph induced by the closed subwalk $v_{\mu} \ldots v_{r} v_{1} v_{2}$ contains a circuit $Q_{4}$ of length at most $r-\mu+2$. So, it is obvious that $r \geq 8$.

Lemma 4.3.3 Let $H$ be a 3-connected graph embedded in a surface $S$. Assume that $e$ is an edge of $H$ incident with faces $f^{\prime}$ and $f^{\prime \prime}$. Then $f^{\prime}=f^{\prime \prime}$ iff there is a non-contractible closed curve $\phi$ in the surface $S$ such that $\phi \cap H$ $(=\phi \cap\{e\})$ is a single point. Furthermore, $f^{\prime}=f^{\prime \prime}$ implies that $d_{H}\left(f^{\prime}\right) \geq 8$ if $H$ is simple and triangle free.

Proof. The first statement is obvious since $H$ is 3-connected. By Lemma 4.3.2 , the second statement holds as $H$ is triangle free and $\delta(H) \geq 3$.

Definition 4.3.4 Let $H$ be a graph embedded in a surface $S$ and $C$ be a given dominating, chordless circuit of $H$. A good face $f$ is normal (with respect to the circuit $C$ ) if $f$ has only one vertex which is not on $C$. A good face $f$ is special (with respect to the circuit $C$ ) if $f$ is not normal.

Examples of normal and special faces are illustrated in the following figures. 


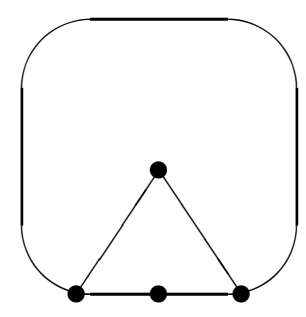

(normal 4-face)

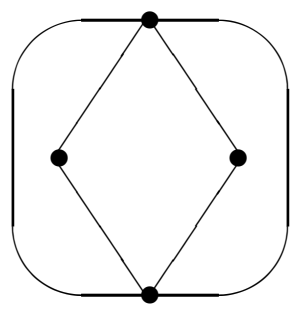

(special 4-face)

For the sake of convenience of later discussion, we list Euler contributions of some edges where $e$ is associated with $\left\{x, y, f^{\prime}, f^{\prime \prime}\right\}$ :

\begin{tabular}{|c|c|c|c|c|}
\hline$d_{H}(x)$ & $d_{H}(y)$ & $d_{H}\left(f^{\prime}\right)$ & $d_{H}\left(f^{\prime \prime}\right)$ & $\Phi(e)$ \\
\hline 3 & 3 & 5 & 5 & $\frac{1}{15}$ \\
\hline 3 & 3 & 5 & 6 & $\frac{1}{30}$ \\
\hline 3 & 3 & 5 & 7 & $\frac{1}{105}$ \\
\hline 3 & 4 & 4 & 5 & $\frac{1}{30}$ \\
\hline 3 & 4 & 4 & 6 & 0 \\
\hline 3 & 4 & 4 & 7 & $-\frac{1}{42}$ \\
\hline 3 & 4 & 5 & 5 & $-\frac{1}{60}$ \\
\hline 3 & 4 & 5 & 6 & $-\frac{1}{20}$ \\
\hline 3 & 4 & 5 & 7 & $-\frac{31}{420}$ \\
\hline 4 & 4 & 4 & 5 & $-\frac{1}{20}$ \\
\hline 4 & 4 & 4 & 6 & $-\frac{1}{12}$ \\
\hline 4 & 4 & 4 & 7 & $-\frac{3}{28}$ \\
\hline 4 & 4 & 5 & 6 & $-\frac{2}{15}$ \\
\hline
\end{tabular}


Chords of Graphs Embedded in Torus and Klein Bottle

\subsection{Proof of the main theorem}

We prove the theorem by contradiction. Our proof consists of four parts. Part one gives some basic structures, part two discusses the existence of positive edges, part three describes five non-avoidable configurations, and part four implements charge-discharge on five non-avoidable configurations.

Let $C=v_{1} v_{2} \cdots v_{m} v_{1}$ be a longest circuit of $G$ without chord.

Let $H$ be a graph obtained from $G$ by contracting each bridge of $C$ into a single vertex.

Part 1. Some basic structures.

(1). $C$ is a longest circuit of $H$, and $C$ is a dominating, chordless circuit of $H$.

(2). $H$ is 3 -connected. For each vertex $x \notin C, d_{H}(x) \geq 4$ since $G$ is 4 connected. And, $d_{H}(x) \geq 3$ for each $x \in V(C)$.

(3). $H$ is triangle free, for otherwise, $C$ can be extended.

(4). $C$ is of length at least 8 since $d_{H}(x) \geq 4$ for every $x \notin V(H)$.

(5). We investigate the local structure around some edges of $C$. Let $e=$ $v_{1} v_{2} \in E(C)$ and $f^{\prime}$ and $f^{\prime \prime}$ be the faces of $H$ on the two sides of $e$ (that is, $e$ is associated with $\left.\left\{v_{1}, v_{2}, f^{\prime}, f^{\prime \prime}\right\}\right)$.

If

$$
\max \left\{d_{H}\left(f^{\prime}\right), d_{H}\left(f^{\prime \prime}\right)\right\} \leq 7 \text { and } d_{H}\left(f^{\prime}\right)+d_{H}\left(f^{\prime \prime}\right) \leq 12,
$$

then we claim that

(5-a). $f^{\prime}$ and $f^{\prime \prime}$ are good faces and therefore, they are distinct. 
If, in addition, both $f^{\prime}$ and $f^{\prime \prime}$ are normal, then we claim that ((5-b), $(5-\mathrm{c}),(5-\mathrm{d}))$

(5-b). $\left|E\left(f^{\prime}\right) \cap E\left(f^{\prime \prime}\right)\right|=1$ (that is, the edge $e=v_{1} v_{2}$ is the only common edge of those two faces.)

(5-c). $V\left(f^{\prime}\right) \backslash V(C) \neq V\left(f^{\prime \prime}\right) \backslash V(C)$.

$(5-\mathrm{d}) \cdot d_{H}\left(f^{\prime}\right)+d_{H}\left(f^{\prime \prime}\right) \geq 10$.

Proof of (5-a): Since $f^{\prime}$ and $f^{\prime \prime}$ are of degree $\leq 7$, by Lemma 4.3.3, their boundaries are circuits and therefore, they are good faces. Since they are good, the edge $e$ cannot be passed twice by the boundary of any one of them, they must be different.

Proof of (5-b): Let $Q^{\prime}$ (and $Q^{\prime \prime}$ ) be the maximal segment of $C$ contained in the boundary of the normal face $f^{\prime}$ (and $f^{\prime \prime}$, respectively). Here, the edge $e \in Q^{\prime} \cap Q^{\prime \prime}$.

Let $P$ be a maximal segment of $C$ contained in $Q^{\prime} \cap Q^{\prime \prime}$. We first claim that the length of $P$ is at most one. If not, then every internal vertex of $P$ must be of degree two since $f^{\prime}$ and $f^{\prime \prime}$ are distinct and are on the two sides of the segment $P$. This contradicts that $\delta(H) \geq 3$ (by (2)).

Thus, we can see that the claim is true if $Q^{\prime} \cap Q^{\prime \prime}$ contains only one segment. So, we assume that $Q^{\prime} \cap Q^{\prime \prime}$ contains more than one segment.

Since $Q^{\prime}, Q^{\prime \prime}$ are contained in the circuit $C, Q^{\prime} \cap Q^{\prime \prime}$ consists of at most two segments, say, $P^{\prime}$ and $P^{\prime \prime}$. Furthermore, $Q^{\prime} \cup Q^{\prime \prime}=C$. Note that, we have already proved that each segment contained in $Q^{\prime} \cap Q^{\prime \prime}$ is of length at most one. So, if the claim is not true, each segment $P^{\prime}$ and $P^{\prime \prime}$ is of length 
precise one. Hence,

$$
\begin{gathered}
|E(C)|=\left|E\left(Q^{\prime}\right)\right|+\left|E\left(Q^{\prime \prime}\right)\right|-\left|E\left(P^{\prime}\right)\right|-\left|E\left(P^{\prime \prime}\right)\right| \\
=\left(d_{H}\left(f^{\prime}\right)-2\right)+\left(d_{H}\left(f^{\prime \prime}\right)-2\right)-2 \\
\quad \leq 12-6=6,
\end{gathered}
$$

This contradicts (4) that $C$ is of length at least 8 .

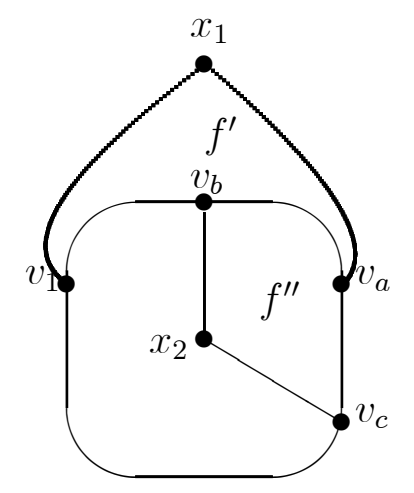

$Q^{\prime} \cap Q^{\prime \prime}$ has 1 segment

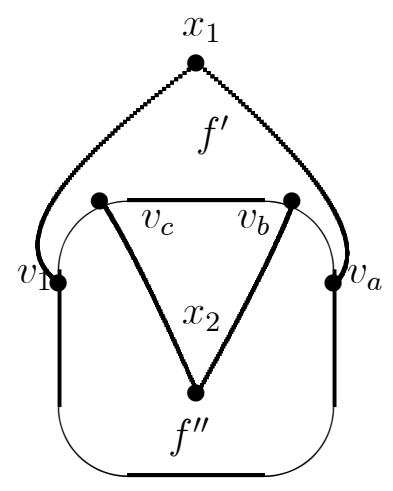

$Q^{\prime} \cap Q^{\prime \prime}$ has 2 segments

Proof of (5-c). Let $x_{1} \in V\left(f^{\prime}\right) \backslash V(C)$ and $x_{2} \in V\left(f^{\prime \prime}\right) \backslash V(C)$. By (5-b), $e=v_{1} v_{2}$ is the only one edge contained in both $f^{\prime}$ and $f^{\prime \prime}$. Therefore, if $x_{1}=x_{2}$, then $x_{1} v_{1} v_{2} x_{1}$ would be a triangle in the triangle-free graph $H$. A contradiction.

Proof of (5-d). Let $f^{\prime}=x_{1} v_{1} C v_{j} x_{1}$ and $f^{\prime \prime}=x_{2} v_{2} \bar{C} v_{h} x_{2}$ since $e=v_{1} v_{2}$ is the only edge in $E\left(f^{\prime}\right) \cap E\left(f^{\prime \prime}\right)$. 
We have another circuit $C^{\prime}=v_{h} x_{2} v_{2} v_{1} x_{1} v_{j} C v_{h}$. Here, since $C$ is a longest circuit in $H$, we have that

$$
\begin{gathered}
|E(C)| \geq\left|E\left(C^{\prime}\right)\right| \\
=|E(C)|-\left|E\left(v_{h} C v_{1}\right)\right|-\left|E\left(v_{2} C v_{j}\right)\right|+\left|E\left(v_{h} x_{2} v_{2}\right)\right|+\left|E\left(v_{1} x_{1} v_{j}\right)\right| \\
=|E(C)|-\left(d_{H}\left(f^{\prime}\right)-3\right)-\left(d_{H}\left(f^{\prime \prime}\right)-3\right)+4 \\
=|E(C)|-d_{H}\left(f^{\prime}\right)-d_{H}\left(f^{\prime \prime}\right)+10 .
\end{gathered}
$$

Hence,

$$
d_{H}\left(f^{\prime}\right)+d_{H}\left(f^{\prime \prime}\right) \geq 10
$$

This proves our claim of (5-d).

(6). Let $f$ be a face of degree 4. It is easy to prove that if $E(f) \cap E(C) \neq \emptyset$, or some vertex of $f$ is of degree 3 , then $f$ must be a normal 4-face.

(7). We investigate the local structure of a degree 3 vertex of $H$. Let $v_{i} \in V(C)$ with $d_{H}\left(v_{i}\right)=3$ and let $v_{i} x \in E(H) \backslash E(C)$. Let $B$ be the bridge of $C$ in the original graph $G$ that the vertex $x$ of $H$ is created by the contraction of $B$, and let $v_{j} \in A(B) \backslash\left\{v_{i}\right\}$.

(7-a). We claim that there is a path in $B$ joining $v_{i}$ and $v_{j}$ of length at least 3.

(7-b). We claim that $\left|E\left(v_{i} C v_{j}\right)\right| \geq 3$ and $\left|E\left(v_{j} C v_{i}\right)\right| \geq 3$

(7-c). We claim that each face of $H$ containing the edge $v_{i} x$ is of degree at least 5 . 
(7-d). We claim that if $e=v_{i} v_{i+1} \in E(C)$ with $d_{H}\left(v_{i}\right)=d_{H}\left(v_{i+1}\right)=3$, then every face containing the edge $e$ is of degree at least 5.

Proof of (7-a). Since $d_{G}\left(v_{i}\right) \geq 4$ and $d_{H}\left(v_{i}\right)=3$, all vertices of $N_{G}\left(v_{i}\right) \backslash$ $V(C)$ are in the same bridge of $C$, say, $B$, and, hence, $|V(B)| \geq 2$ in $G$. Let $V(B)=\left\{b_{1}, b_{2}, \ldots, b_{t}\right\}(t \geq 2)$. Note that $d_{B}\left(v_{i}\right) \geq 2$ in $B$ since $d_{G}\left(v_{i}\right) \geq 4$. Without loss of generality, let $v_{i} b_{1}, v_{i} b_{2} \in E(G)$. For $v_{j} \in A(B) \backslash\left\{v_{i}\right\}$, let $v_{j} b_{k} \in E(G)$ for some $k(1 \leq k \leq t)$, there are some paths joining $v_{i}$ and $v_{j}$ in $B$ since $B \backslash A(B)$ is connected. Let $P_{v_{i} v_{j}}$ be a longest path of $B$ joining $v_{i}$ and $v_{j}$ in $B$. We claim that $\left|E\left(P_{v_{i} v_{j}}\right)\right| \geq 3$. Otherwise, $\left|E\left(P_{v_{i} v_{j}}\right)\right| \leq 2$ implies $\left|E\left(P_{v_{i} v_{j}}\right)\right|=2$. Let $P_{v_{i} v_{j}}=v_{i} b_{k} v_{j}$ and let $b_{1} \in\left\{b_{1}, b_{2}\right\} \backslash\left\{b_{k}\right\}$ as $d_{B}\left(v_{i}\right) \geq 2$. There is a path $P^{\prime}$ of $B \backslash A(B)$ joining $b_{1}$ and $b_{k}$ since $B \backslash A(B)$ is connected. The path $v_{i} b_{1} P^{\prime} b_{k} v_{j}$ would be longer than $P_{v_{i} v_{j}}$, a contradiction.

Proof of (7-b). It is obvious by (7-a) (see the following figure).

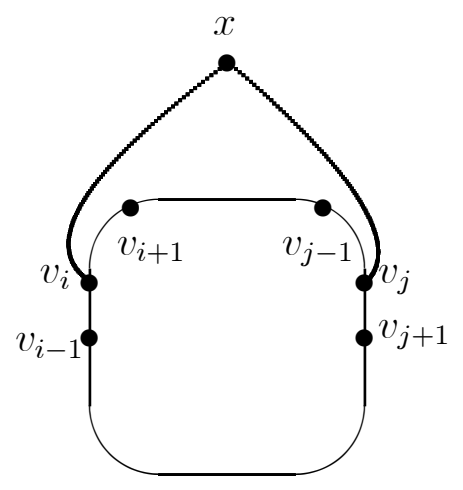

Figure for $(7-b)$ 
Proof of (7-c). Assume that there is a face $f$ of degree 4 where $f$ is incident with the edge $v_{i} x$. We can see that $f$ is neither a normal 4 -face (by (7-b)), nor a special 4-face (by $(6)$ since $d_{H}\left(v_{i}\right)=3$ ).

Proof of (7-d): Let $v_{i} x, v_{i+1} y \in E(H) \backslash E(C)$. By (7-c), each face incident with either $v_{i} x$ or $v_{i+1} y$ is of degree at least 5. Assume that there is a face $f$ that contains the edge $e=v_{i} v_{i+1}$ but not any of $v_{i} x$ and $v_{i+1} y$. Since $d_{H}\left(v_{i}\right)=d_{H}\left(v_{i+1}\right)=3$, the face $f$ must use the segment $v_{i-1} v_{i} v_{i+1} v_{i+2}$ of $C$. So, the face $f$ must be of length at least 5 .

(8). Let edge $e=v_{i} v_{i+1} \in C$ be associated with $\left\{v_{i}, v_{i+1}, f^{\prime}, f^{\prime \prime}\right\}$ and $f^{\prime} \neq f^{\prime \prime}$. We claim that (8-a). if both $f^{\prime}$ and $f^{\prime \prime}$ are normal faces, then

$$
\left\{d_{H}\left(f^{\prime}\right), d_{H}\left(f^{\prime \prime}\right)\right\} \neq\{4,4\} \text { or }\{4,5\}
$$

(8-b). if both $f^{\prime}$ and $f^{\prime \prime}$ are normal faces and $d_{H}\left(v_{i}\right)=d_{H}\left(v_{i+1}\right)=3$, then

$$
\left\{d_{H}\left(f^{\prime}\right), d_{H}\left(f^{\prime \prime}\right)\right\} \neq\{5,5\} \text { or }\{5,6\} \text {. }
$$

Proof of (8-a): (8-a) is an immediate corollary of (5-d).

Illustration of (8-b) are in the following figures.

Proof of (8-b): Assume that $d_{H}\left(v_{i}\right)=d_{H}\left(v_{i+1}\right)=3$ and $\left\{d_{H}\left(f^{\prime}\right), d_{H}\left(f^{\prime \prime}\right)\right\}$ $=\{5,5\}$. By (5-b), we know that $E\left(f^{\prime}\right) \cap E\left(f^{\prime \prime}\right)=\left\{e=v_{i} v_{i+1}\right\}$. Let $f^{\prime}=w v_{i} v_{i+1} v_{i+2} v_{i+3} w, f^{\prime \prime}=x v_{i-2} v_{i-1} v_{i} v_{i+1} x$ where $x, w \notin V(C)$ and $w \neq x$ (by $(5-\mathrm{c}))$. By $(7-\mathrm{a})$, there is a path $P_{(i-2)(i+1)}$ of length at least 3 in a bridge $B_{i+1}$ of $G$ joining $v_{i-2}$ and $v_{i+1}$ where $B_{i+1}$ is the bridge incident with $v_{i+1}$, and there is a path $P_{i(i+3)}$ of length at least 3 of $B_{i}$ joining $v_{i}$ and $v_{i+3}$ where $B_{i}$ 


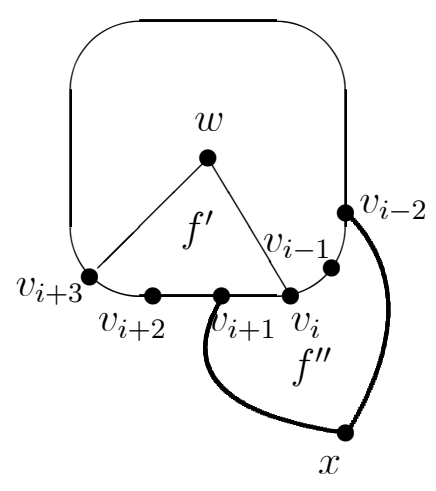

(In $H$ )

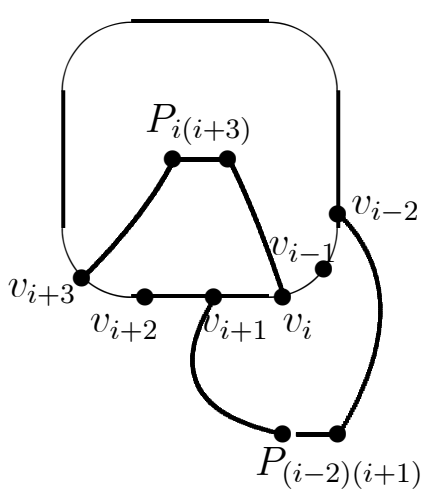

$(\operatorname{In} G)$

is the bridge incident with $v_{i}$. Then the circuit $v_{i-2} P_{(i-2)(i+1)} v_{i+1} v_{i} P_{i(i+3)} v_{i+3}$ $C v_{i-2}$ would be longer than $C$. This contradicts that $C$ is a longest circuit of $H$.

Similarly, if $\left\{d_{H}\left(f^{\prime}\right), d_{H}\left(f^{\prime \prime}\right)\right\}=\{5,6\}$, then $C$ would not be a longest circuit of $H$, either.

Part Two. The existence of positive edges.

An edge $e$ of $H$ is positive, negative or zero if $\Phi(e)>0,<0$, or $=0$, respectively.

(9). We claim that every nonnegative edge $e$ is incident with two distinct faces.

Assume that $e$ is incident with only one face $f$. Since $H$ is triangle free and $\delta(H) \geq 3$, then $d_{H}(f) \geq 8$ by Lemma 4.3.2. We have that

$$
\Phi(e) \leq \frac{1}{3}+\frac{1}{3}+\frac{1}{8}+\frac{1}{8}-1<0
$$

(10). We claim that there exists some positive edge in $H$. 
We prove it by contradiction. If the claim is false, then $\Phi(e)=0$ for every edge $e \in E(H)$ since $\sum_{e \in E(H)} \Phi(e)=0$.

There are only three possibilities:

$$
\begin{array}{ll}
\text { or } \quad & \Phi(e)=\frac{1}{3}+\frac{1}{4}+\frac{1}{4}+\frac{1}{6}-1=0 \\
\text { or } & \Phi(e)=\frac{1}{4}+\frac{1}{4}+\frac{1}{4}+\frac{1}{4}-1=0 \\
& \Phi(e)=\frac{1}{3}+\frac{1}{3}+\frac{1}{6}+\frac{1}{6}-1=0
\end{array}
$$

since $d_{H}(x) \geq 3$ and $H$ has no triangles.

Note that $(* * *)$ does not happen for any edge $e \in E(H) \backslash E(C)$ since $d_{H}(y) \geq 4$ for every $y \notin V(C)$.

(10.1) We claim that every vertex of $V(C)$ is of degree 4.

Assume that $d_{H}(x)=3$ for some vertex $x$ of $H$. By $(2), x \in V(C)$.

Let $e=x y \in E(H) \backslash E(C)(x \in V(C), y \notin V(C))$, let $f^{\prime}$ and $f^{\prime \prime}$ be the faces on the two sides of edge $e=x y$. Since $d_{H}(x)=3$ then we must have the case $(*)$ here:

$\Phi(e)=\frac{1}{d_{H}(x)}+\frac{1}{d_{H}(y)}+\frac{1}{d_{H}\left(f^{\prime}\right)}+\frac{1}{d_{H}\left(f^{\prime \prime}\right)}-1=\frac{1}{3}+\frac{1}{4}+\frac{1}{4}+\frac{1}{6}-1=0\left(^{*}\right)$ as $d_{H}(x)=3$ and $d_{H}(y) \geq 4$.

(10.1.1) We claim that the case $\left(^{*}\right)$ will not happen. Assume that the case $\left(^{*}\right)$ holds for an edge $e \in E(H) \backslash E(C)$, then there is at least one 4-face incident with $e$. This contradicts (7-c) since $d_{H}(x)=3$ and the face $f^{\prime}$ contains the edge calculation : xy. 
Chords of Graphs Embedded in Torus and Klein Bottle

(10.1.2) By (10.1.1), only the case (**) holds for every $e=x y \in E(H) \backslash$ $E(C)$.

The case $\left({ }^{* *}\right)$ implies $d_{H}(x)=4$ for all $x \in V(C)$ since each vertex of $V(C)$ is incident with at least one edge of $E(H) \backslash E(C)$. So, by (10.1.1) and $(10.1 .2), d_{H}(x)=4$ for every $x \in V(C)$.

(10.2) Now we consider edges in $E(C)$. Let $e\left(=v_{1} v_{2}\right) \in C$ be associated with $\left\{v_{1}, v_{2}, f^{\prime}, f^{\prime \prime}\right\}$, by $(10.1), d_{H}\left(v_{1}\right)=d_{H}\left(v_{2}\right)=4$, we must have

$$
\Phi(e)=\frac{1}{d_{H}\left(v_{1}\right)}+\frac{1}{d_{H}\left(v_{2}\right)}+\frac{1}{d_{H}\left(f^{\prime}\right)}+\frac{1}{d_{H}\left(f^{\prime \prime}\right)}-1=\frac{1}{4}+\frac{1}{4}+\frac{1}{4}+\frac{1}{4}-1=0
$$

as $H$ is triangle free. So, $d_{H}\left(f^{\prime}\right)=d_{H}\left(f^{\prime \prime}\right)=4$. Obviously, both 4 -faces $f^{\prime}$ and $f^{\prime \prime}$ must be normal faces by (6). But, this contradicts (8-a). Hence, there exists at least one edge with non-zero Euler contribution. By the equation (4) of Section 4.2, there are some positive edges.

(11). We claim that every positive edge must be on the longest circuit $C$.

By (10), there is some positive edge. Assume that our claim is false. Let $e=w v \in E(H) \backslash E(C)$ be a positive edge associated with $\left\{v, w, f^{\prime}, f^{\prime \prime}\right\}$ and $v \in V(C), w \notin V(C)$.

Notice that $d_{H}(v) \geq 3, d_{H}(w) \geq 4$ by $(2)$.

(11.1). We claim that $d_{H}(v)=3$. If not, assume $d_{H}(v) \geq 4$, then

$$
\Phi(e)=\frac{1}{d_{H}(v)}+\frac{1}{d_{H}(w)}+\frac{1}{d_{H}\left(f^{\prime}\right)}+\frac{1}{d_{H}\left(f^{\prime \prime}\right)}-1 \leq \frac{1}{4}+\frac{1}{4}+\frac{1}{4}+\frac{1}{4}-1 \leq 0
$$

as $d_{H}(w) \geq 4$ and $H$ being triangle-free, a contradiction. 
(11.2). We claim that $d_{H}\left(f^{\prime}\right), d_{H}\left(f^{\prime \prime}\right) \geq 5$.

By (11.1), we have that $d_{H}(v)=3$. Therefore, the claim follows immediately by $(7-c)$.

By (11.1) and (11.2), we have that

$$
\Phi(e) \leq \frac{1}{3}+\frac{1}{4}+\frac{1}{5}+\frac{1}{5}-1<0,
$$

This contradicts that $e$ is a positive edge.

Part three. Five unavoidable configurations.

(12). We investigate the local structure of the face(s) incident with a positive edge $e$.

By (11), each positive edge $e$ must be on the longest circuit $C$. By (10), every edge not on $C$ is a non-positive edge. Without loss of generality, let $e \in E(C)$ be a positive edge associated with $\left\{v_{1}, v_{2}, f^{\prime}, f^{\prime \prime}\right\}$.

If both $d_{H}\left(v_{1}\right), d_{H}\left(v_{2}\right) \geq 4$ or one of them is of at least 6 , then

$$
\Phi(e)=\frac{1}{d_{H}\left(v_{1}\right)}+\frac{1}{d_{H}\left(v_{2}\right)}+\frac{1}{d_{H}\left(f^{\prime}\right)}+\frac{1}{d_{H}\left(f^{\prime \prime}\right)}-1 \leq 0,
$$

since both $d_{H}\left(f^{\prime}\right), d_{H}\left(f^{\prime \prime}\right) \geq 4$. So,

$$
\min \left\{d_{H}\left(v_{1}\right), d_{H}\left(v_{2}\right)\right\}=3 \text { and } \max \left\{d_{H}\left(v_{1}\right), d_{H}\left(v_{2}\right)\right\} \leq 5
$$

Thus ,

$$
\left\{d_{H}\left(v_{1}\right), d_{H}\left(v_{2}\right)\right\} \in\{\{3,3\},\{3,4\},\{3,5\}\}
$$

where we assume that $d_{H}\left(v_{1}\right) \leq d_{H}\left(v_{2}\right)$.

(12.1) We first investigate the case $d_{H}\left(v_{1}\right)=3$ and $d_{H}\left(v_{2}\right) \geq 4$. That is,

$$
\left\{d_{H}\left(v_{1}\right), d_{H}\left(v_{2}\right)\right\}=\{3,4\} \text { or }\{3,5\} \text {. }
$$


(a).

$$
\min \left\{d_{H}\left(f^{\prime}\right), d_{H}\left(f^{\prime \prime}\right)\right\} \geq 4
$$

since $H$ is triangle-free.

$$
\max \left\{d_{H}\left(f^{\prime}\right), d_{H}\left(f^{\prime \prime}\right)\right\} \leq 5,
$$

for otherwise, $\Phi(e) \leq 0$. If $\left\{d_{H}\left(f^{\prime}\right), d_{H}\left(f^{\prime \prime}\right)\right\}=\{5,5\}$, then $\Phi(e)<0$. So,

$$
\left\{d_{H}\left(f^{\prime}\right), d_{H}\left(f^{\prime \prime}\right)\right\}=\{4,4\} \text { or }\{5,4\} .
$$

(b). By (a), one of $f^{\prime}, f^{\prime \prime}$ is a 4 -face. The 4 -face, say, $f^{\prime \prime}$ must be normal by (6). And note that at least one of $f^{\prime}, f^{\prime \prime}$ is special (by (8-a)). Hence, only one case left by (a) and (b):

$$
\begin{aligned}
& \left\{d_{H}\left(f^{\prime}\right), d_{H}\left(f^{\prime \prime}\right)\right\}=\{5,4\} \text { where } f^{\prime} \text { is a special } 5 \text {-face, } f^{\prime \prime} \text { is a } \\
& \text { normal 4-face and }\left\{d_{H}\left(v_{1}\right), d_{H}\left(v_{2}\right)\right\}=\{3,4\} \text { or }\{3,5\} \\
& \text { (call it Configuration 1). }
\end{aligned}
$$

(12.2) We, then, investigate the case that $d_{H}\left(v_{1}\right)=d_{H}\left(v_{2}\right)=3$.

By $(7-\mathrm{d})$, none of $\left\{f^{\prime}, f^{\prime \prime}\right\}$ is a $4-$ face. So, $d_{H}\left(f^{\prime}\right), d_{H}\left(f^{\prime \prime}\right) \geq 5$.

If both $d_{H}\left(f^{\prime}\right), d_{H}\left(f^{\prime \prime}\right)$ are at least 6 , or one of $\left\{d_{H}\left(f^{\prime}\right), d_{H}\left(f^{\prime \prime}\right)\right\}$ is at least 8 , then $\Phi(e) \leq 0$. So, we have

$$
\min \left\{d_{H}\left(f^{\prime}\right), d_{H}\left(f^{\prime \prime}\right)\right\}=5 \text { and } \max \left\{d_{H}\left(f^{\prime}\right), d_{H}\left(f^{\prime \prime}\right)\right\} \leq 7 .
$$

Therefore, there are only 3 possible subcases left in this case:

$$
\left\{d_{H}\left(f^{\prime}\right), d_{H}\left(f^{\prime \prime}\right)\right\} \in\{\{5,5\},\{5,6\},\{5,7\}\} .
$$


(a). By (8-b), we have that $f^{\prime}$ and $f^{\prime \prime}$ cannot be both normal faces if $\left\{d_{H}\left(f^{\prime}\right), d_{H}\left(f^{\prime \prime}\right)\right\}=\{5,5\}$ or $\{5,6\}$.

(b). We claim that $f^{\prime}$ and $f^{\prime \prime}$ cannot be both special if $\left\{d_{H}\left(f^{\prime}\right), d_{H}\left(f^{\prime \prime}\right)\right\}=$ $\{5,5\}$ or $\{5,6\}$. Otherwise, assume both $f^{\prime}$ and $f^{\prime \prime}$ are special. Since $f^{\prime}$ is a special 5-face, the boundary of $f^{\prime}$ must be $v_{1} v_{2} x v_{i} y v_{1}$ for some $x, y \notin V(C)$ and $v_{i} \in V(C)$. Thus, the boundary of the face $f^{\prime \prime}$ must use the segment $v_{m} v_{1} v_{2} v_{3}$ of $C$ since $d_{H}\left(v_{1}\right)=d_{H}\left(v_{2}\right)=3$. This would imply that $d_{H}\left(f^{\prime \prime}\right) \geq 7$ if $f^{\prime \prime}$ is special.

From the discussion above, we know that, except for the case that

$$
\left\{d_{H}\left(f^{\prime}\right), d_{H}\left(f^{\prime \prime}\right)\right\}=\{5,7\}
$$

the positive edge $e$ is incident with precisely one special face. Let $f^{\prime}$ be the special face if only one of $\left\{f^{\prime}, f^{\prime \prime}\right\}$ is special, or $f^{\prime}$ is the shorter one if both $f^{\prime}$ and $f^{\prime \prime}$ are special, $f^{\prime}$ is the longer one if both $f^{\prime}$ and $f^{\prime \prime}$ are normal.

Above discussion lead us to the following Configurations:

$$
\begin{aligned}
& d_{H}\left(f^{\prime}\right)=5 \text { and } d_{H}\left(f^{\prime \prime}\right) \in\{6,7\} \text { (where } f^{\prime} \text { is special) and } d_{H}\left(v_{1}\right) \\
& \left.=d_{H}\left(v_{2}\right)=3 \text { (call it Configuration } 2\right) \text {. } \\
& d_{H}\left(f^{\prime}\right) \in\{6,7\} \text { and } d_{H}\left(f^{\prime \prime}\right)=5 \text { (where } f^{\prime} \text { is special and } f^{\prime \prime} \text { is } \\
& \text { normal) and } \left.d_{H}\left(v_{1}\right)=d_{H}\left(v_{2}\right)=3 \text { (call it Configuration } 3\right) . \\
& d_{H}\left(f^{\prime}\right)=d_{H}\left(f^{\prime \prime}\right)=5 \text { (where } f^{\prime} \text { is special, and } f^{\prime \prime} \text { is normal) and } \\
& \left.d_{H}\left(v_{1}\right)=d_{H}\left(v_{2}\right)=3 \text { (call it Configuration } 4\right) \text {. }
\end{aligned}
$$




$$
\begin{aligned}
& d_{H}\left(f^{\prime}\right)=7 \text { and } d_{H}\left(f^{\prime \prime}\right)=5 \text { and both } f^{\prime} \text { and } f^{\prime \prime} \text { are normal and } \\
& d_{H}\left(v_{1}\right)=d_{H}\left(v_{2}\right)=3(\text { call it Configuration } 5) .
\end{aligned}
$$

\section{Part 4. Charge-discharge.}

(13). From the previous subsections, we have found that the graph $H$ embedded in the torus or the Klein bottle must have some positive edges and they must locate along the longest circuit $C$.

Let $\psi: E(H) \rightarrow R$ be a function such that $\psi(e)=\Phi(e)$, the Euler contribution (as the initial charge). As we already knew in Section 4.2,

$$
\sum_{e \in E(H)} \psi(e)=0
$$

In this subsection, we will re-distribute (charge and discharge, as commonly called) the function $\psi$ such that the total sum of the function $\psi(e)$ remains the same as before, and we will show later that, under the new function, the total sum of the function will be negative. This will contradict to equation $(\mathrm{A})$.

For the sake of convenience of later discussion, we define some terms. Under the function $\psi=\Phi$, an edge $e$ with $\psi(e)>0$ is called a $D$-edge (means that this edge will be discharged later). We notice that, in the configurations 1 to 4 , a $D$-edge is incident with a special face $f^{\prime}$, which is called a $C$ - $D$-face (it means that the charge-discharge operation will occur along the edges of this face). We also notice that, in the configuration 5 , the faces $f^{\prime}, f^{\prime \prime}$ incident with the $D$-edge $e$ are normal, which, are called $C-D$-faces, in this case. 
Each $e \notin E(C)$, is called a $C$-edge, A $C$-edge $e$ is called a [++]-edge if it is incident with two $C-D$-faces, or is a $[+-]$-edge if it is incident with at most one $C-D$-face (it means that the edge $e$ will be charged twice from two sides or charged at most once from one side).

The charge-discharge operation is described as follows:

For a $C-D$-face $f^{\prime}$ in the configurations 1 to 4 , we need to show that

$$
\sum_{e \in E(f)} \epsilon(e) \psi(e)<0
$$

where $\epsilon(e)=1$ if $e$ is a $D$-edge or $e$ is a $[+-]$-edge, and $\epsilon(e)=\frac{1}{2}$ if $e$ is a $[++]$-edge. (That is, each edge $e \in E(f) \backslash\{\mathrm{D}$-edges $\}$, which is of negative value originally, will be charged with $\epsilon(e)|\psi(e)|$ from those $D$-edges along the face $f^{\prime}$. And we will show that, those $[++]$ and $[+-]$-edges remain nonpositive, and those $D$-edges will be of negative value after the operation.) For the configuration 5 , let $e=v_{1} v_{2}$ be the $D$-edge and let $v_{2} u$ and $v_{1} z$ be the edges of $E(H) \backslash E(C)$ that are incident with $e=v_{1} v_{2}$. The charge-discharge operation occurs only among the edges $\in\left\{v_{1} v_{2}, v_{2} u, v_{1} z\right\}$ as follows: each $e^{*} \in\left\{v_{1} z, v_{2} u\right\}$ will be charged $\frac{1}{2}\left|\psi\left(e^{*}\right)\right|$ from the $D$-edge $v_{1} v_{2}$.

Note that the configuration 5 is very different that no face is special, it will be dealt with separately. And the configuration 4 needs a little more attention since a rough estimation would not lead us to a negative total value of $\psi$ around the special face. In the subsections (13-1), and (13-2), we will deal with the first four configurations. 
Chords of Graphs Embedded in Torus and Klein Bottle

(13-1) The total value of $\psi(e)$ for all $e \in E\left(f^{\prime}\right) \cap E(C)$ for configurations $1-4$.

Since $f^{\prime}$ is a special face of degree at most 7 and contains at least one edge $e=v_{1} v_{2}$ of $C$, the face has exactly four edges in $E\left(f^{\prime}\right) \backslash E(C)$ and $d_{H}\left(f^{\prime}\right)-4$ edges of $E(C)$. Since no edge of $E(H) \backslash E(C)$ is positive (by (11)), they are $C$-edges. Considering the worst case in calculations, each edge of $E\left(f^{\prime}\right) \cap E(C)$ could be positive (therefore, a $D$-edge).

(i). If the degree of the special face $f^{\prime}$ is 5 , then $e=v_{1} v_{2}$ is the only edge of $E\left(f^{\prime}\right) \cap E(C)$. Hence,

(i-1). for the configuration 1 ,

$$
\sum_{e \in E\left(f^{\prime}\right) \cap E(C)} \psi(e)=\psi\left(v_{1} v_{2}\right) \leq \frac{1}{3}+\frac{1}{4}+\frac{1}{5}+\frac{1}{4}-1=\frac{1}{30} ;
$$

(i-2). for the configuration 2 ,

$$
\sum_{e \in E\left(f^{\prime}\right) \cap E(C)} \psi(e)=\psi\left(v_{1} v_{2}\right) \leq \frac{1}{3}+\frac{1}{3}+\frac{1}{5}+\frac{1}{6}-1=\frac{1}{30} ;
$$

(i-3). for the configuration 4 ,

$$
\sum_{e \in E\left(f^{\prime}\right) \cap E(C)} \psi(e)=\psi\left(v_{1} v_{2}\right) \leq \frac{1}{3}+\frac{1}{3}+\frac{1}{5}+\frac{1}{5}-1=\frac{1}{15} .
$$

(ii). For the configuration 3, let $e=v^{\prime} v^{\prime \prime} \in E\left(f^{\prime}\right) \cap E(C)$ be associated with $\left\{v^{\prime}, v^{\prime \prime}, f^{\prime}, f^{*}\right\}$. By applying (7-c) and (7-d) whenever there is a possibility that a degree 3 vertex or a pair of degree 3 vertices is involved, the sequence of degrees $\left\{d_{H}\left(v^{\prime}\right), d_{H}\left(v^{\prime \prime}\right), d_{H}\left(f^{\prime}\right), d_{H}\left(f^{*}\right)\right\}$ must be one of following cases:

$$
\left\{3,3, d_{H}\left(f^{\prime}\right), \geq 5\right\},\left\{3, \geq 4, d_{H}\left(f^{\prime}\right), \geq 4\right\},\left\{\geq 4, \geq 4, d_{H}\left(f^{\prime}\right), \geq 4\right\} .
$$


Hence,

$$
\begin{aligned}
& \psi(e) \leq \\
& \leq \max \left\{\frac{1}{3}+\frac{1}{3}+\frac{1}{d_{H}\left(f^{\prime}\right)}+\frac{1}{5}-1, \frac{1}{3}+\frac{1}{4}+\frac{1}{d_{H}\left(f^{\prime}\right)}+\frac{1}{4}-1, \frac{1}{4}+\frac{1}{4}+\frac{1}{d_{H}\left(f^{\prime}\right)}+\frac{1}{4}-1\right\} \\
&= \frac{1}{3}+\frac{1}{3}+\frac{1}{d_{H}\left(f^{\prime}\right)}+\frac{1}{5}-1=\frac{1}{d_{H}\left(f^{\prime}\right)}-\frac{2}{15} .
\end{aligned}
$$

So,

$$
\begin{gathered}
\sum_{e \in E\left(f^{\prime}\right) \cap E(C)} \psi(e) \leq\left[d_{H}\left(f^{\prime}\right)-4\right] \cdot\left[\frac{1}{d_{H}\left(f^{\prime}\right)}-\frac{2}{15}\right] \\
\leq[6-4] \cdot\left[\frac{1}{6}-\frac{2}{15}\right]=\frac{1}{15}
\end{gathered}
$$

(since $d_{H}\left(f^{\prime}\right)=6$ or 7 ).

(13-2) The total value of $\psi(e)$ for all $e \in E\left(f^{\prime}\right) \backslash E(C)$ for configurations $1-4$.

We notice that the special face $f^{\prime}$ has exactly four edges in $E\left(f^{\prime}\right) \backslash E(C)$.

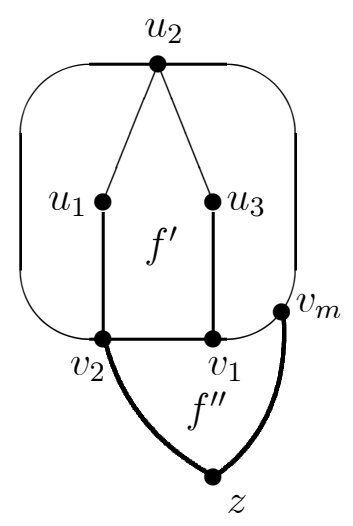

Configuration 1 
(i). For the configuration 1 , the degree of $f^{\prime}$ is 5 . Let $f^{\prime}=v_{1} v_{2} u_{1} u_{2} u_{3} v_{1}$ where $u_{1}, u_{3} \notin V(C)$. Here, $d_{H}\left(v_{1}\right)=3$ and $d_{H}\left(v_{2}\right) \geq 4$. Then the degree sequence $\left\{d_{H}\left(v_{1}\right), d_{H}\left(u_{3}\right), d_{H}\left(f^{\prime}\right), d_{H}\left(f^{*}\right)\right\}$ associated with the edge $v_{1} u_{3}$ must be $\{3, \geq 4,5, \geq 5\}$ by (2) and (7-c); and the degree sequence $\left\{d_{H}(x), d_{H}(y), d_{H}\left(f^{\prime}\right), d_{H}\left(f^{*}\right)\right\}$ associated with the edge $x y \in\left\{v_{2} u_{1}, u_{1} u_{2}\right.$, $\left.u_{2} u_{3}\right\}$ must be $\{\geq 4, \geq 4,5, \geq 4\}$ by (2) and (3). Thus,

$$
\psi\left(v_{1} u_{3}\right) \leq \frac{1}{3}+\frac{1}{4}+\frac{1}{5}+\frac{1}{5}-1=-\frac{1}{60},
$$

and

$$
\psi\left(v_{2} u_{1}\right), \psi\left(u_{2} u_{1}\right), \psi\left(u_{2} u_{3}\right) \leq \frac{1}{4}+\frac{1}{4}+\frac{1}{5}+\frac{1}{4}-1=-\frac{1}{20} .
$$

So, the total $\psi$ value of those $C$-edges is

$$
\sum_{e \in E\left(f^{\prime}\right) \backslash E(C)} \psi(e) \leq-\frac{1}{60}+3 \cdot\left(-\frac{1}{20}\right)=-1 / 6
$$

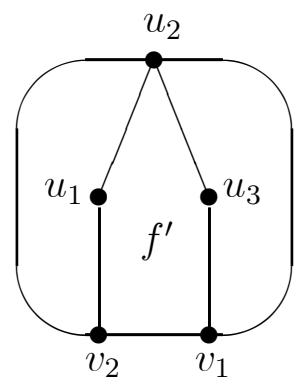

Configuration 2

(ii). For the configurations 2 and 4 , the degree of $f^{\prime}$ is 5 . Let $f^{\prime}=$ $v_{1} v_{2} u_{1} u_{2} u_{3} v_{1}$ where $u_{1}, u_{3} \notin V(C)$. Then the degree sequence $\left\{d_{H}(x), d_{H}(y)\right.$, $\left.d_{H}\left(f^{\prime}\right), d_{H}\left(f^{*}\right)\right\}$ associated with the edge $x y \in\left\{v_{2} u_{1}, v_{1} u_{3}\right\}$ must be $\{3, \geq$ $4,5, \geq 5\}$ by $(2)$ and $(7-c)$; and the degree sequence $\left\{d_{H}(x), d_{H}(y), d_{H}\left(f^{\prime}\right)\right.$, 
$\left.d_{H}\left(f^{*}\right)\right\}$ associated with the edge $x y \in\left\{u_{1} u_{2}, u_{2} u_{3}\right\}$ must be $\{\geq 4, \geq 4,5, \geq$ $4\}$ by (2) and (3). Thus,

$$
\psi\left(v_{2} u_{1}\right), \psi\left(v_{1} u_{3}\right) \leq \frac{1}{3}+\frac{1}{4}+\frac{1}{5}+\frac{1}{5}-1=-\frac{1}{60},
$$

and

$$
\psi\left(u_{1} u_{2}\right), \psi\left(u_{2} u_{3}\right) \leq \frac{1}{4}+\frac{1}{4}+\frac{1}{5}+\frac{1}{4}-1=-\frac{1}{20} .
$$

So, the total $\psi$ value of those $C$-edges is

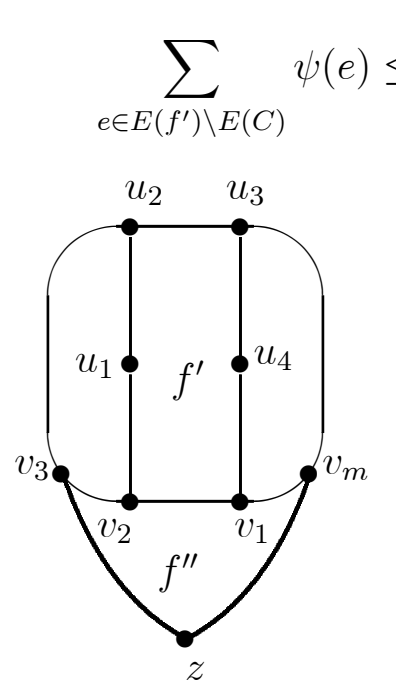

Configuration 3-1

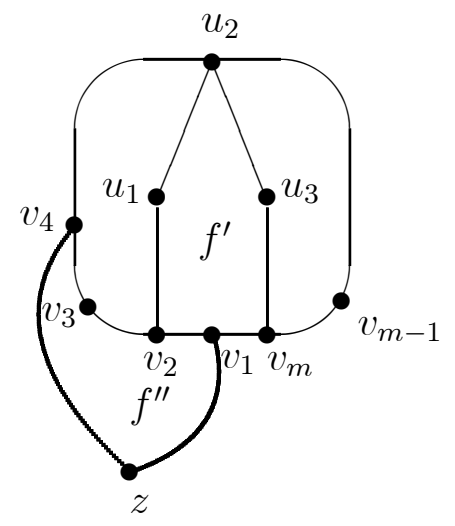

Configuration 3-2

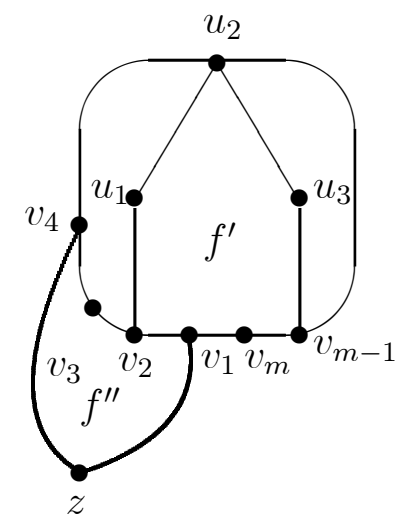

Configuration 3-5 


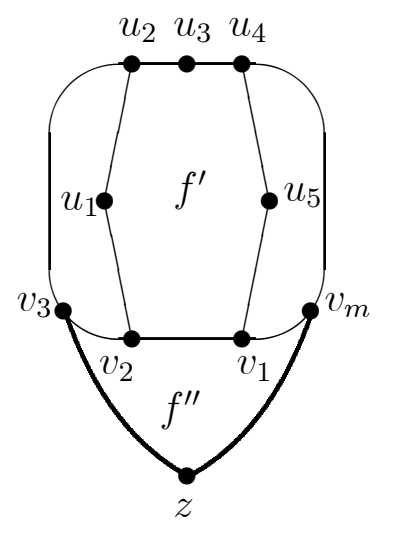

Configuration 3-3

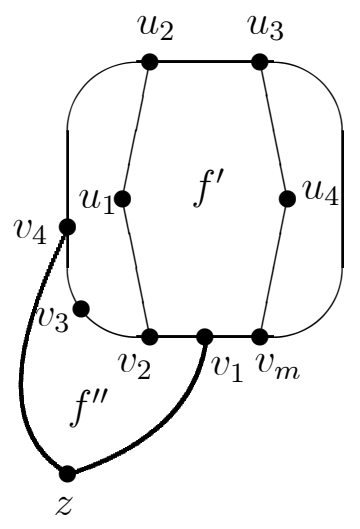

Configuration 3-4

(iii). For the configuration 3, the degree of $f^{\prime}$ is 6 or 7 . Let $e=v^{\prime} v^{\prime \prime} \in$ $E\left(f^{\prime}\right) \backslash E(C)$ be associated with $\left\{v^{\prime}, v^{\prime \prime}, f^{\prime}, f^{*}\right\}$. By applying (7-c) whenever there is a possibility that a degree 3 vertex is involved, the sequence of degrees $\left\{d_{H}\left(v^{\prime}\right), d_{H}\left(v^{\prime \prime}\right), d_{H}\left(f^{\prime}\right), d_{H}\left(f^{*}\right)\right\}$ must be one of following cases:

$$
\left\{3, \geq 4, d_{H}\left(f^{\prime}\right), \geq 5\right\},\left\{\geq 4, \geq 4, d_{H}\left(f^{\prime}\right), \geq 4\right\}
$$

Hence,

$$
\begin{aligned}
\psi(e) & \leq \\
& \leq \max \left\{\frac{1}{3}+\frac{1}{4}+\frac{1}{d_{H}\left(f^{\prime}\right)}+\frac{1}{5}-1, \frac{1}{4}+\frac{1}{4}+\frac{1}{d_{H}\left(f^{\prime}\right)}+\frac{1}{4}-1\right\} \\
& =\frac{1}{3}+\frac{1}{4}+\frac{1}{d_{H}\left(f^{\prime}\right)}+\frac{1}{5}-1 \leq \frac{1}{d_{H}\left(f^{\prime}\right)}-\frac{13}{60} \leq \frac{1}{6}-\frac{13}{60}=-\frac{1}{20} .
\end{aligned}
$$

So,

$$
\sum_{e \in E\left(f^{\prime}\right) \backslash E(C)} \psi(e) \leq 4\left[-\frac{1}{20}\right]=-\frac{1}{5}
$$

(13-3). By the calculations in (13-1) and (13-2), we are ready for estimating $\sum_{e \in E\left(f^{\prime}\right)} \epsilon(e) \psi(e)$ for some configurations. 
Note that, by considering the worst case in the calculation, each $C$-edge should be considered as a $[++]$-edge. That is, the coefficient $\epsilon$ will be $\frac{1}{2}$ for the worst cases in the estimation.

(i). For configuration 1 (see (13-1)-(i-1) and (13-2)-(i)), we have that

$$
\begin{gathered}
\sum_{e \in E\left(f^{\prime}\right)} \epsilon(e) \psi(e) \leq \sum_{e \in E\left(f^{\prime}\right) \cap E(C)} \epsilon(e) \psi(e)+\sum_{e \in E\left(f^{\prime}\right) \backslash E(C)} \epsilon(e) \psi(e) \\
\leq\left(\frac{1}{30}\right)+\left(\frac{1}{2}\right)\left(-\frac{1}{6}\right)=-\frac{1}{20}
\end{gathered}
$$

(ii). For configuration 2 (see (13-1)-(i-2) and (13-2)-(ii)), we have that

$$
\begin{aligned}
\sum_{e \in E\left(f^{\prime}\right)} \epsilon(e) \psi(e) & \leq \sum_{e \in E\left(f^{\prime}\right) \cap E(C)} \epsilon(e) \psi(e)+\sum_{e \in E\left(f^{\prime}\right) \backslash E(C)} \epsilon(e) \psi(e) \\
& \leq\left(\frac{1}{30}\right)+\left(\frac{1}{2}\right)\left(-\frac{2}{15}\right)=-\frac{1}{30}
\end{aligned}
$$

(iii). For configuration 3,(see (13-1)-(ii) and (13-2)-(iii)), we have that

$$
\begin{aligned}
\sum_{e \in E\left(f^{\prime}\right)} \epsilon(e) \psi(e) \leq & \sum_{e \in E\left(f^{\prime}\right) \cap E(C)} \epsilon(e) \psi(e)+\sum_{e \in E\left(f^{\prime}\right) \backslash E(C)} \epsilon(e) \psi(e) \\
& \leq\left(\frac{1}{15}\right)+\left(\frac{1}{2}\right)\left(-\frac{1}{5}\right)=-\frac{1}{30}
\end{aligned}
$$

The value of $\sum_{e \in E\left(f^{\prime}\right)} \epsilon(e) \psi(e)$ for each configuration 1, 2 and 3 is negative. However, the same estimation for the configuration 4 would give us a zero. This is not what we would like to have. Of course, we notice that the estimations in (13-1) and (13-2) are not very tight at all. Therefore, some further attention is needed for the configuration 4 . 
(13-4) For configuration 4, the degree of both faces $f^{\prime}$ (special) and $f^{\prime \prime}$ (normal) is 5 and $d_{H}\left(v_{1}\right)=d_{H}\left(v_{2}\right)=3$, where $u_{1}, u_{3} \notin V(C)$. Here, $d_{H}\left(v_{1}\right)=$ $d_{H}\left(v_{2}\right)=3$.

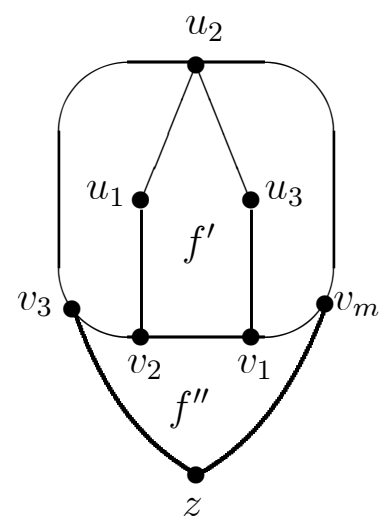

Configuration 4

Let $f^{\prime}$ and $f_{u_{3} v_{1}}$ be the faces on the two sides of edge $u_{3} v_{1}$. By (7-c), $d_{H}\left(f_{u_{3} v_{1}}\right) \geq 5$ since $d_{H}\left(v_{1}\right)=3$.

Case 4-1. If $d_{H}\left(f_{u_{3} v_{1}}\right) \geq 6$.

For the $D$-edge $e=v_{1} v_{2}$,

$$
\psi\left(v_{1} v_{2}\right)=\frac{1}{3}+\frac{1}{3}+\frac{1}{5}+\frac{1}{5}-1=\frac{1}{15} .
$$

Note that $d_{H}\left(f_{u_{3} v_{1}}\right) \geq 6$,

$$
\psi\left(u_{3} v_{1}\right) \leq \frac{1}{3}+\frac{1}{4}+\frac{1}{5}+\frac{1}{6}-1=-\frac{1}{20} .
$$

By the earlier results (see (13-1) and (13-2)), we have that

$$
\psi\left(u_{1} u_{2}\right), \psi\left(u_{2} u_{3}\right) \leq-\frac{1}{20}
$$

and

$$
\psi\left(v_{2} u_{1}\right) \leq-\frac{1}{60} .
$$


So, for all the $C$-edges, we have that

$$
\sum_{e \in E\left(f^{\prime}\right) \backslash E(C)} \psi(e) \leq-\left[\frac{1}{60}+\frac{1}{20}+\frac{1}{20}+\frac{1}{20}\right]=-\frac{10}{60} .
$$

Hence,

$$
\sum_{e \in E\left(f^{\prime}\right)} \epsilon(e) \psi(e) \leq \frac{1}{2}\left(-\frac{10}{60}\right)+\frac{1}{15}<0
$$

Case 4-2. If $d_{H}\left(f_{u_{3} v_{1}}\right)=5$.

(a). We shall determine that the $C$-edge $u_{3} v_{1}$ must be a [+-]-edge (thus, the coefficient $\epsilon$ would be 1 instead of $\frac{1}{2}$ ).

Assume that $u_{3} v_{1}$ is a $[++]$-edge, hence, $f_{u_{3} v_{1}}$ is a $C-D$-face with respect to some edges of $E\left(f_{u_{3} v_{1}}\right) \cap E(C)$.

First, we show that $f_{u_{3} v_{1}}$ cannot be normal. Assume that the $C-$ $D$-face $f_{u_{3} v_{1}}$ is normal. Thus, the normal 5 -face $f_{u_{3} v_{1}}$, has the boundary $v_{1} u_{3} v_{m-2} v_{m-1} v_{m} v_{1}$. By (7-a), there is a path $P$ of $G$ joining $v_{m-2}$ and $v_{1}$ of length at least 3 , where $E(P) \cap E(C)=\emptyset$. Then $v_{m-2} P v_{1} v_{m} z v_{3} C v_{m-2}$ (see the figure of Configuration 4) would be longer than $C$, a contradiction. So, the face $f_{u_{3} v_{1}}$ must be special.

Since $f_{u_{3} v_{1}}$ is special and $d_{H}\left(v_{1}\right)=3, v_{m} v_{1}$ is the only edge in $E\left(f_{u_{3} v_{1}}\right) \cap$ $E(C)$. Furthermore, $d_{H}\left(v_{m}\right) \geq 4$.

Now, with a calculation, we have that $\psi\left(v_{m} v_{1}\right)<0$. Since $v_{m} v_{1}$ is the only edge in $E\left(f_{u_{3} v_{1}}\right) \cap E(C)$, the face $f_{u_{3} v_{1}}$ cannot be a $C-D$-face and therefore, it proves our claim that $u_{3} v_{1}$ is a [+-]-edge. 
Chords of Graphs Embedded in Torus and Klein Bottle

(b). Calculations: For the $D$-edge $v_{1} v_{2}$,

$$
\psi\left(v_{1} v_{2}\right) \leq \frac{1}{3}+\frac{1}{3}+\frac{1}{5}+\frac{1}{5}-1=\frac{1}{15}
$$

Now, take a half charge of all other $C$-edges and total charge of the [+-]-edge $u_{3} v_{1}$, we have that

$$
\begin{aligned}
\sum_{e \in E\left(f^{\prime}\right)} \epsilon(e) \psi(e) & \left.=\psi\left(v_{1} v_{2}\right)+\frac{1}{2}\left[\psi\left(v_{2} u_{1}\right)+\psi\left(u_{1} u_{2}\right)\right)+\psi\left(u_{2} u_{3}\right)\right]+\psi\left(u_{3} v_{1}\right) \\
& =\frac{1}{15}-\frac{1}{2}\left[\frac{1}{60}+\frac{1}{20}+\frac{1}{20}\right]-\frac{1}{60}=\frac{1}{15}-\frac{9}{120}<0 .
\end{aligned}
$$

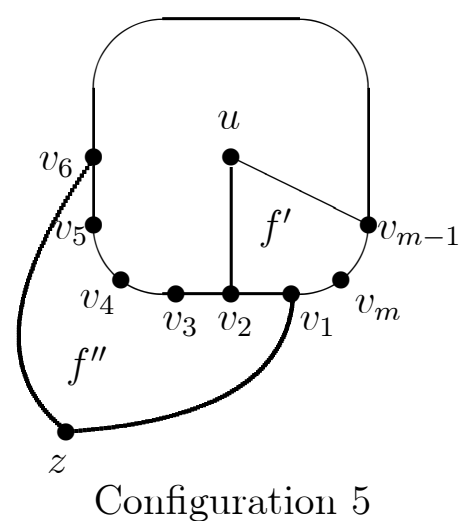

(14). Configuration 5. $\left\{d_{H}\left(f^{\prime}\right), d_{H}\left(f^{\prime \prime}\right)\right\}=\{5,7\}$ where $f^{\prime}$ is a normal 5 -face and $f^{\prime \prime}$ is a normal 7 -face.

By (5-b), $E\left(f^{\prime}\right) \cap E\left(f^{\prime \prime}\right)=e\left(=v_{1} v_{2}\right)$, without loss of generality, let $f^{\prime}=v_{1} v_{2} u v_{m-1} v_{m} v_{1}$ (where $u \notin V(C)$ ) and $f^{\prime \prime}=z v_{1} v_{2} \cdots v_{6} z$ (where $z \notin V(C))$. 
Note that the face $f^{\prime}$ (or, $f^{\prime \prime}$ as well) is not a $C-D$-face for any edge of $E\left(f^{\prime}\right) \cap E(C)$ with respect to any configration 1-4 since the $C-D$-faces for configrations 1-4 must be special. Thus, if the charge-discharge orperation occurs more than once in the face $f^{\prime}$ (or, similar for $f^{\prime \prime}$ ), it must be for the $D$-edge $v_{m-1} v_{m}$ or $\left(v_{5} v_{6}\right)$ in another configuration 5 . There is no conflict in the culculation here since the charge-discharge operation occurs only at incident $C$-edges for the configuration 5 . Let the edge $v_{1} z$ be associate with $\left\{v_{1}, z, f^{\prime \prime}, f_{v_{1} z}\right\}$ and the edge $v_{2} u$ be associate with $\left\{v_{2}, u, f^{\prime}, f_{v_{2} u}\right\}$. By (7-c), $d_{H}\left(f_{v_{1} z}\right) \geq 5, d_{H}\left(f_{v_{2} u}\right) \geq 5$ since $d_{H}\left(v_{1}\right)=d_{H}\left(v_{2}\right)=3$.

So, we have following calculation:

$$
\begin{gathered}
\psi\left(v_{1} v_{2}\right)=\frac{1}{3}+\frac{1}{3}+\frac{1}{5}+\frac{1}{7}-1=\frac{1}{105} . \\
\psi\left(v_{2} u\right) \leq \frac{1}{3}+\frac{1}{4}+\frac{1}{5}+\frac{1}{5}-1=-\frac{1}{60} \\
\psi\left(v_{1} z\right) \leq \frac{1}{3}+\frac{1}{4}+\frac{1}{5}+\frac{1}{7}-1=-\frac{31}{420}, \\
\psi\left(v_{1} z\right)+\psi\left(v_{2} u\right) \leq-\frac{38}{420} .
\end{gathered}
$$

Therefore,

$$
\sum_{e \in E\left(f^{\prime}\right)} \epsilon(e) \psi(e) \leq \psi\left(v_{1} v_{2}\right)+\frac{1}{2} \psi\left(v_{1} z\right)+\frac{1}{2} \psi\left(v_{2} u\right)<0 .
$$

Final conclusion. After charging-discharging, we have seen that the total charge of all the edges is negative. This contradicts to the fact $\sum_{e \in E(H)} \psi(e)=0$ (equation (4) of Section 4.2). 


\subsection{Remarks}

The proof of the main theorem actually shows that every longest circuit of a 4-connected graph embedded in a surface with a nonnegative characteristic has a chord. However, it is already known that every 4-connected graph embedded in a sphere or projective plane (Tutte [10] and [12]) is hamiltonian as is every 5-connected toroidal graph ([13]). But it remains open (Grünbaum [5] and Nash-Williams [7]) that whether 4-connected toroidal graph is hamiltonian. 


\section{Bibliography}

[1] Alspach, B. and Godsil, C.D., Cycles in Graphs, Ann. Discrete Math. 27 (1985).

[2] Arnold, B.H., Intuitive concepts in elementary topology, Prentice-Hall, Inc., Englewood Cliffs, N.J. (1962).

[3] Fleischner,H. and Stiebitz,M., A solution to a colouring problem, Discrete Math.101(1992),39-48.

[4] Fleischner,H., Uniqueness of Maximal Dominating Cycles in 3-Regular Graphs and of Hamiltonian Cycles in 4-Regular Graphs. J. Graph Theory, 18(1994),449-459.

[5] Grünbaum, B., Polytopes, graphs, and complexes, Bull. Amer Math. Soc. 76(1970), 1131-1201.

[6] Lebesgue, H., Quelques conséquences simples de la formule d'Euler, $J$. de Math., 9, Sér. 19, (1940), pp. 27-43

[7] Nash-Williams, C. St. J. A., Unexplored and semi-explored territories in graph theory, in "New Directions in Graph Theory,"pp. 169-176, Academic Press, New York, (1973). 
[8] Saaty, T.L., The Four Color Problem, McGraw-Hill, (1977). Trans. Amer. Math. Soc. 82 (1956). 99-116.

[9] Sanders,D.P., and Zhao, Y., A Note on the Three Color Problem. Graphs and Combinatorics (1995).

[10] Tutte, W.T., A theorem on planar graphs. Trans. Amer. Math. Soc. 82 (1956). 99-116.

[11] Thomason, A.C., Hamiltonian cycles and Uniquely edge colourable graphs. Annals of Discrete Mathematics. 3(1978),259-268.

[12] Thomas, R., and Yu, X., 4-connected projective graph is Hamilton. J. Combin. Theory Ser. B., 62(1994),114-132.

[13] Thomas, R., and Yu, X., 5-connected Toroidal graph are Hamiltonian. J. Combin. Theory Ser. B., 69(1997),79-96.

[14] Thomassen, C., Chords of longest circuit in cubic graphs. J. Combin. Theory Ser. B., 71(1997),215-230.

[15] Thomassen, C., Independent Dominating Sets and a Second Hamilton Cycle in Regular Graphs. J. Combin. Theory Ser. B., 72(1998),104-109.

[16] Thomason,A.G., Hamilton circuits and uniquely edge colourable graphs, Ann. Discrete Math.3(1978),259-268.

[17] Zhang, C.-Q., Longest circuits and their Chords. J. Graph Theory, 11 (1987), 521-529. 